\title{
Broad Band Shock Associated Noise Predictions in Axisymmetric and Asymmetric Jets Using an Improved Turbulence Scale Model
}

\author{
Anuroopa Kalyan* and Sergey A. Karabasov ${ }^{\dagger}$ \\ Queen Mary University of London, Mile End Road, London E1 4NS, United Kingdom
}

Supersonic jets that are subject to off-design operating conditions are marked by three distinct regions in their far-field spectra: mixing noise, screech and Broadband Shock Associated Noise (BBSAN). BBSAN is conspicuous by the prominent multiple peaks. The Morris and Miller BBSAN model that is based on an acoustic analogy, offering a straightforward implementation for RANS, forms the foundation of the present work. The analogy model robustly captures the peak frequency noise, that occurs near Strouhal number of about 1 , based on the nozzle exit diameter but leads to major sound under prediction for higher frequencies. In the jet mixing noise literature, it has been shown that an inclusion of frequency dependence into the characteristic length and temporal scales of the effective noise sources improves the far-field noise predictions. In the present paper, several modifications of the original Morris and Miller model are considered that incorporate the frequency dependent scales as recommended in the jet mixing noise literature. In addition to these, a new mixed scale model is proposed that incorporates a correlation scale that depends both on the mean-flow velocity gradient and the standard mixing noise-type scaling based on the dissipation of turbulent kinetic energy. In comparison with the original Morris and Miller model, the mixed scale model shows considerable improvements in the noise predictions for the benchmark axisymmetric convergent-divergent and convergent jets. Further to this validation, the new model has been applied for improved predictions for elliptic jets of various eccentricity. It has been shown that,

\footnotetext{
* Doctoral Researcher, School of Engineering and Material Sciences, a.kalyan@qmul.ac.uk

${ }^{\dagger}$ Reader in Computational Modelling, School of Engineering and Material Sciences, s.karabasov@qmul.ac.uk
} 
for the same thrust conditions, the elliptical nozzles lead to noise reduction at the source in comparison with the baseline axisymmetric jets.

\section{Nomenclature}

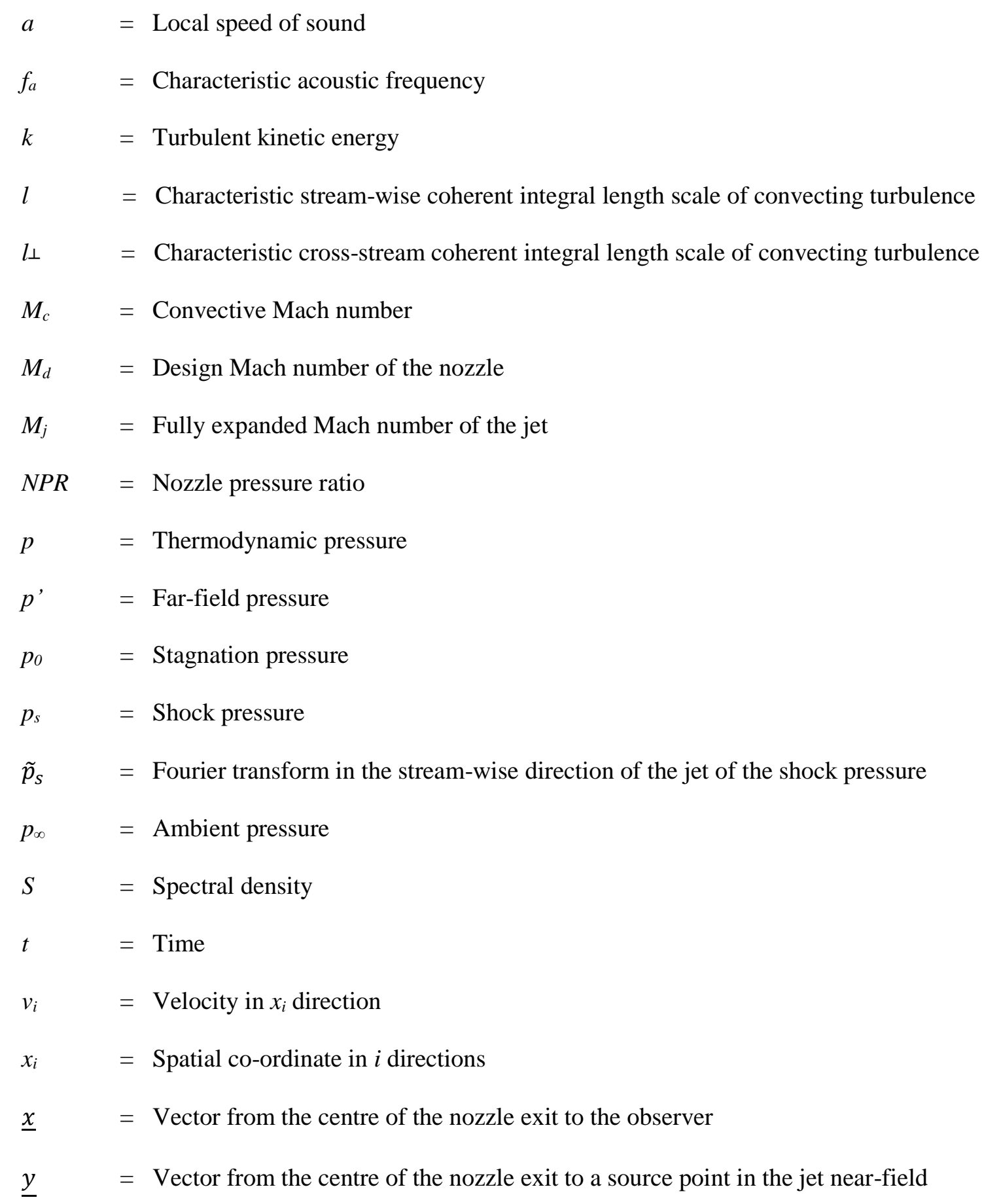




$$
\begin{array}{ll}
\alpha & =\text { Mixed Scale Model Parameter } \\
\gamma & =\text { Ratio of specific heats of an ideal gas } \\
\kappa & =\text { Wavenumber } \\
\eta & =\text { Vector between two points in the source region } \\
\rho_{\infty} & =\text { Free-stream density } \\
\pi & =\text { Logarithmic expression of pressure } \\
\pi_{g} & =\text { Component of vector Green's function } \\
\tau & =\text { Retarded time } \\
\omega & =\text { Radial frequency } \\
\omega_{c} & =\text { Characteristic frequency } \\
\text { Subscripts } & \\
i, j & =
\end{array}
$$

\section{Introduction}

Broadband Shock Associated Noise (BBSAN) is an important component of supersonic jets that operate at off-design conditions which occur when the pressure at the nozzle exit is not equal to the ambient pressure. This mismatch is overcome by the jet by undergoing a series of expansions and compressions that are conspicuously visible as quasi-periodic shock cells. Since the noise sources of BBSAN are primarily located in the regions where these shock cells interact with the turbulent eddies in the shear layer that originate from the lip of the nozzle, they are visible on the far-field spectra prominently as multiple peaks, closely associated with the dominant shock cells. 
BBSAN is recognised as a growing cause of concern in civil and military jets, as cabin noise at high altitudes, and during jet launches from sea vessels, respectively. For producing optimal engineering solutions of future jet exhaust systems with reduced BBSAN, cost-effective noise prediction schemes are required, especially those which are based on fast-turn-around Reynolds Averaged Navier Stokes (RANS) modelling. Such schemes would be particularly relevant with respect to new propulsion airframe aeroacoustics studies, which combine aerodynamic and aeroacoustic predictions of highly integrated engines with the airframe, such as those explored by Bridges et al. [1].

The first prediction methodologies for BBSAN were developed by Harper-Bourne and Fisher [2]. Further advancements in prediction methods was due by Tam and Tanna [3] and Tam [4] who also included jet heating effects, in addition to accounting for the effect of the slow jet spreading and turbulence dissipation on shock cells in the analysis. In comparison with the Harper- Bourne and Fisher model [2], the latter models are much more theory grounded as compared to the earlier work: they employed stochastic modelling to describe the effect of turbulence and the Pack-Prandtl theory [5] to model the shock cell structure. As a further development, Morris and Miller [6] proposed a BBSAN prediction scheme that is made specific to nozzle geometry by considering the development of the jet flow, including modelling of internal flow in the nozzle with the use of a two-equation RANS solution. The model is advantageous in the sense that it only requires the knowledge of two parameters that govern the jet flow, nozzle pressure ratio (NPR) and the total temperature ratio (TTR). The two inlet boundary conditions, together with the specified nozzle geometry and ambient flow conditions fully describe the flow inside the nozzle and in the exterior jet. This is the model which is chosen as a baseline approach for the current work.

For coupling acoustic modelling with the flow solution, the Morris and Miller model [6] uses the triple scale flow solution decomposition in the framework of the classical Lighthill acoustic analogy approach [7]. In the acoustic analogy model, the flow is decomposed into the steady mean-flow solution, turbulence fluctuations, and acoustic fluctuations. The governing Navier-Stokes equations are then re-arranged leaving just the linear wave propagation operator on the left-hand-side of the equations while the rest of the nonlinear terms including all non-uniform flow terms are grouped on the right-hand-side. For BBSAN, which 
predominantly propagates at polar angles close to $90^{\circ}$ to the jet, in case of unheated jets, only the leading order noise source term is retained that is be due to the interaction of turbulent eddies with mean-flow velocity gradient due to the shock cell structure. The effective noise source at the far-field then corresponds to a two-point two-time velocity covariance function that includes both turbulent velocity fluctuation and the mean-flow velocity gradient. The covariance function needed for BBSAN description is more complex than the second-order velocity auto-correlation functions that are relatively routinely measured in experiments or obtained from eddy-resolving computations. Hence, the first step of the Morris and Miller source model [6] is to factor out the effect of spatially variable mean-flow gradient from the effective source function to reduce the source function to the conventional second-order velocity auto-correlation function form which is further simplified by taking into account sound radiating in the far-field observer direction. Following the jet noise literature, e.g. Tam and Auriault [8], the velocity auto-correlation function is then modelled assuming an analytical Gaussian-exponential shape function which is fully described by the corresponding correlation scales and the amplitude. The last conceptual step of the model is to evaluate the correlation scales and the amplitude by linearly scaling the characteristic turbulence scales obtainable from a RANS solution with some constant calibration parameters that are typically evaluated from matching the model predictions to the far-field noise data. For instance, for a $k$-epsilon RANS model, the characteristic space and time scales can be linked to the corresponding RANS turbulent kinetic energy and the energy dissipation rate distributions as implemented in the original model by Morris and Miller [6] and their model implementation by Kalyan and Karabasov [9].

In the present work, the validity of the last conceptual step of the Morris and Miller model [6], i.e. the simple linear scaling procedure to reconstruct the effective acoustic source scales from the RANS-type characteristic turbulence dissipation scales is going to be revised. The motivation for the current revision came from the fact that, despite the ability of the Morris and Miller model [6] to reasonably accurately predict the peak frequency noise, which corresponds to the Strouhal number based on the nozzle diameter at $S t_{D} \sim 1$, it leads to major under-predictions at higher frequencies. This discrepancy escalates to notable differences in Over All Sound Pressure Levels (OASPL) and thus calls for improvement. 
The first direction of investigation considered in the current paper deals with incorporating several frequency-dependent lengths scale models into the original Morris and Miller model [6], which used frequency-independent correlation scales. This is motivated by studies in the jet mixing noise literature which showed that incorporation of frequency dependence for the source length scale is consistent with the experimental observations and leads to improved predictions of the far-field sound. The investigation of frequency dependence of the length scale was first performed in the experimental investigation by HarperBourne [10], who suggested an empirical relation such that the length scale is approximately constant at low frequencies and has a nearly inverse dependence on $S t_{D}$ for high frequencies. This formed the basis for Self [11] who developed an analytical model that includes a dependence of the correlation length of the source on the shear layer width and relates the moving axis timescale for turbulent velocity and the length scale. The model relies on accurate definition of the shear layer width that may not be straightforward for complex jets. Self and Basetti [12] further improved their length scale model by introducing another empirical relationship with the assumption that the moving axis timescale is a function only of frequency and axial location in the jet, even though there can be a radial variation associated with the jet velocity. Another model of the same authors suggests defining the correlation length scales with respect to the local turbulent kinetic energy and dissipation rate, similar to the Morris and Miller model [6]. Further examples of frequency-dependent length scale models in the literature include the model by Page et al. [13], which derives its length and time scales from the combinations of local mean velocity, turbulent kinetic energy and dissipation rate. Morris and Boluriaan [14] proposed their own frequency-dependent scale model where the frequency dependence relation was built on the scaling suggested by Harper-Bourne [10]. A similar relation was built into the frequency-dependent scale model used by Leib and Goldstein [15]. More recently, Miller [16] proposed a new frequency-dependent length scale model that is based on the works of Morris and Zaman [17], Morris and Boluriaan [14], and Leib and Goldstein [15]. Several of these models will be implemented in the current work in the attempt to improve high-frequency predictions of the original Morris and Miller model [6]. 
Although the idea of frequency-dependent correlation scales is driven by physics, the majority of the models developed were specifically derived for jet mixing noise. Hence, it remains questionable how suitable the frequency-dependent length scale models, which were calibrated for jet mixing noise, are for BBSAN. In particular, the jet mixing noise mechanism is driven by the fourth-order velocity autocorrelation functions and the velocity correlation functions which are relevant for BBSAN, are secondorder. But the second-order and the fourth-order velocity auto-correlation functions don't share the same functional behaviour. For example, in accordance with the experimental data, e.g. Morris and Zaman [17], the second-order velocity auto-correlation functions have prominent negative loops with amplitude of about $20 \%$ of the peak value at large separations as compared to the fourth-order correlations which remain positive within $2-3 \%$.

Another point to consider when adopting some of the jet mixing noise source features for BBSAN is that a distinct feature of shock-associated noise, is the presence of shock cells in the flow. Hence, the correlation length scales important for BBSAN can be expected to contain a strongly correlated velocity component due to the well-organised shock structure as opposed to the much more random nature of jet mixing noise at large polar angles to the jet flow, which are of interest for BBSAN. Recently, Tan et al. [18] investigated the second-order correlation length scales obtained from high-resolution Particle Image Velocimetry (PIV) measurements for several under-expanded jets issuing from an axisymmetric convergent nozzle. To separate the random fluctuating part from the total velocity fluctuations, the latter were decomposed into the correlated part that contains most of the energy and the uncorrelated part using Proper Orthogonal Decomposition (POD). The two sets of velocity fluctuations, the total and the random parts, were used for obtaining the corresponding integral turbulence scales.

Figure 1 shows some outcomes of the study of Tan et al. [18] that are relevant to the current discussion. The axial length scale distributions corresponding to the second-order velocity auto-correlation function of the vertical velocity component is plotted along the shear layer and in the jet shear layer location. The vertical velocity component corresponds to the effective noise source of the Morris and Miller model [6] at $90^{\circ}$ polar angle to the jet flow. The jet corresponds to NPR $=3.4$ and perfectly expanded $M_{j}=1.45$. These 
conditions are similar to the convergent nozzle jet case (NPR $=3.67$ and $\left.M_{j}=1.5\right)$ that was originally considered by Morris and Miller [6] and which will also be considered in the present work. The difference between the correlation scale based on the total velocity fluctuation, which includes the effect of coherent fluctuations due to the presence of shock cells (both BBSAN and screech in their case) and the scale based on purely random velocity fluctuations is especially striking at the jet centreline, where the shock cells are strongest. It also remains notable in the shear layers where the turbulence intensity is highest. As shown by Tan et al. [18], in contrast to the correlation scale based on the total velocity fluctuations, the correlation scale based on the random velocity field component exhibits a very similar scaling based on the shear layer thickness to the ones previously reported in the literature for supersonic jet cases without significant shock cells, i.e. typical of pure jet mixing noise, e.g. in Fleury et al. [19].

(a)

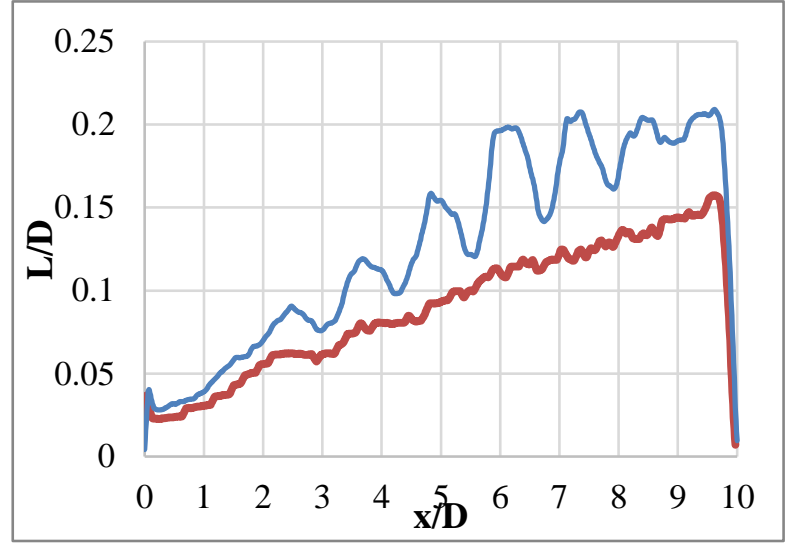

(b)

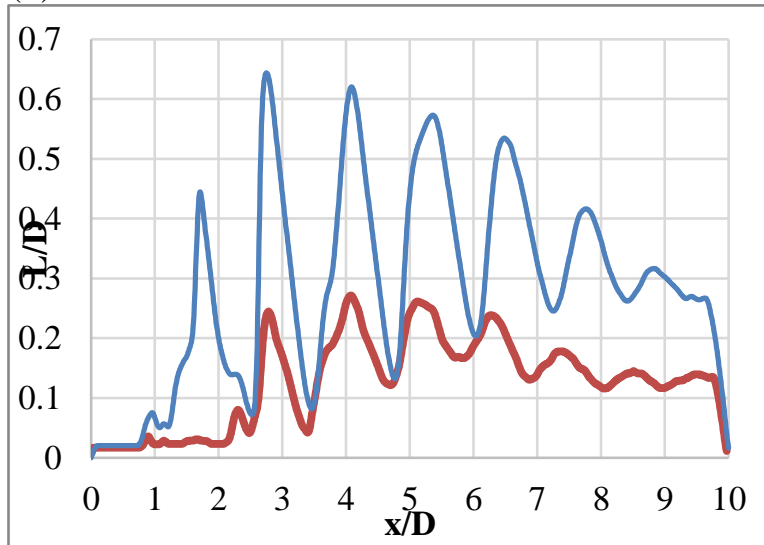

Fig. 1 Length scale comparisons for convergent nozzle of $M_{j}=1.45$ as obtained by Tan et al. [18] along (a) centre of shear layer and (b) jet axis.

The importance of the correlated part of the total velocity fluctuations for shock associated noise due to the presence of strong shock cells as compared to the more random fluctuations typical of pure jet mixing noise suggests that the definition of BBSAN-relevant correlation length scale should include not only the characteristic scale of the turbulence dissipation but also a second scale that is linked to the shock-cell 
structure. For example, the axial mean-flow velocity gradient associated with the shock cell strength can be a valid choice for such a second scale.

The idea of combining two different flow length scales, one based on the turbulence dissipation and the other based on the mean-flow velocity gradient within a single acoustic source model, is not new in the jet noise literature. For example, Frendi et al. [20] considered two time scales, one associated with mean-flow velocity strain and the other with the dissipation of turbulent kinetic energy in application to jet mixing noise. The implementation of their model is not very straightforward due to the need to identify and demarcate different regions of the jet flow where one or the other scale is activated. For example, such demarcation may be impossible to perform accurately at small polar angles to the jet flow, where sound mean flow propagation plays a significant role for jet mixing noise. However, for BBSAN, which is most important for angles around $90^{\circ}$ to the jet, where the sound mean-flow propagation plays a secondary role, the idea of combining the two distinctly different scales under a single suitably modified noise source model of Morris and Miller [6] seems very appealing. Such an idea would address the experimentally observed behaviour of shock-associated noise, that is partly correlated because of the well-organised shock-cell structure and partly random because of turbulence dissipation. It is the implementation of this idea in the new mixed scale model which is the main novelty of the present work.

The paper is organised as the following. In section 2, the mathematical framework of BBSAN model of Morris and Miller [6] is briefly outlined as a starting point for the new models developed. The new models correspond to several extensions of the original Morris and Miller model [6], which implement suggestions from the literature for frequency-dependent correlation scales and also include the new mixed scale model. The mixed scale model uses a frequency-independent scale that is composed of the turbulent energy dissipation scale and the mean-flow velocity gradient scale within a single acoustic source. Implementation details of the models are discussed in Sections 3. Section 4 is devoted to axisymmetric jet modelling where the various BBSAN models implemented are analysed in detail and compared with the experimental data available. Section 5 extends the current acoustic modelling to asymmetric jets by considering several elliptic 
jet flows of varying eccentricity. The results of acoustic modelling of elliptic jets by the new mixed scale model and by the original Morris and Miller model [6] are then discussed, which is followed by conclusions.

\section{Mathematical Model}

To put the current work in perspective, main steps of the BBSAN model of Morris and Miller [6] are briefly outlined below. The starting point of the model is uniform flow acoustic propagation equations where the rest of the non-linear terms of the governing Navier-Stokes equations are grouped on the righthand-side, which follows from the solution decomposition into the steady mean flow, turbulent fluctuations and acoustic fluctuations. By retaining only the leading order (linear) terms of fluctuations, ignoring the viscous and heat conduction effects, and agglomerating the effect of acoustic fluctuations inside the turbulence fluctuations in the remaining source terms, the following governing system of acoustic equations is obtained:

$$
\begin{gathered}
\frac{\partial \pi^{\prime}}{\partial t}+\bar{v}_{j} \frac{\partial \pi^{\prime}}{\partial x_{j}}+\frac{\partial v_{i}^{\prime}}{\partial x_{i}}=\theta \\
\frac{\partial v_{i}^{\prime}}{\partial t}+\bar{v}_{j} \frac{\partial v_{i}^{\prime}}{\partial x_{j}}+v_{j}^{\prime} \frac{\partial v_{i}^{\prime}}{\partial x_{j}}+\bar{a}^{2} \frac{\partial \pi^{\prime}}{\partial x_{i}}=f_{i}^{v}+f_{i}^{a}
\end{gathered}
$$

In the above equations, prime represents the acoustic fluctuation quantities $\pi^{\prime}$ and $v_{i}^{\prime}$. $\theta$ represents the dilatation rate caused by the interaction between the gradients of pressure due to the shock cells and the turbulent velocity fluctuations:

$$
\theta=-v_{s j} \frac{\partial \pi_{t}}{\partial x_{j}}-v_{t j} \frac{\partial \pi_{s}}{\partial x_{j}}
$$


$f_{i}^{v}$ characterises the unsteady force per unit volume associated with interactions between the turbulent velocity fluctuations and the velocity fluctuations associated with the shock cells:

$$
f_{i}^{v}=-v_{s j} \frac{\partial v_{t i}}{\partial x_{j}}-v_{t j} \frac{\partial v_{s i}}{\partial x_{j}}
$$

$f_{i}^{a}$ is the source that represents the unsteady force per unit volume related to the interaction of fluctuations in sound speed, e.g. due to the temperature variation caused by the turbulence, the shock cells, and the associated pressure gradients:

$$
f_{i}^{a}=-a_{s}^{2} \frac{\partial \pi_{t}}{\partial x_{i}}-a_{t}^{2} \frac{\partial \pi_{s}}{\partial x_{i}}
$$

It is further assumed that for unheated jets, which are also of interest in the present paper, one can retain only the turbulence/mean-flow gradient source term (3b). This is because the dilatation rate source (3a) is expected to scale similarly and its effect can be agglomerated in the same source, while (3b) and the source term $(3 \mathrm{c})$ related to entropy generation is expected to be small for unheated jets.

To obtain the acoustic pressure at the far-field for polar angles close to $90^{\circ}$ to the jet, which are relevant for BBSAN, the above acoustic propagation equations are solved analytically using the free-space Green's function technique. The resulting expression for sound power spectral density at the far-field includes a Fourier image of the space-time auto-covariance function of the source $f_{i}^{v}$ that combines a mixture of the turbulent velocity fluctuation and the mean-flow velocity gradient terms (3b). By further assuming that the source function scales such that $f_{i}^{v} \sim p_{s} v_{t} / \rho_{\infty} a_{\infty} l$, where, $p_{s}$ is the shock cell strength, $v_{t}$ is the characteristic turbulent velocity fluctuation and, using the Proudman form for the cross-correlation as well as assuming the isotropy of the source term, the sound power spectral density expression is obtained, 


$$
S(\underline{x}, \omega)=\frac{\omega^{2}}{16 \pi^{2} a_{\infty}^{4} x^{2}} \int_{-\infty}^{\infty} \ldots \int_{-\infty}^{\infty} \frac{1}{l^{2}} p_{s}(\underline{y}) p_{s}(\underline{y}+\eta) R^{v}(\underline{y, \eta}, \tau) \exp \left[i \omega\left(\tau-\frac{\underline{x} \cdot \eta}{x a_{\infty}}\right)\right] d \tau d \eta d \underline{y}
$$

where, $\eta=\underline{z}-\underline{y}$ and $\tau=\tau_{2}-\tau_{1}$ with $\eta=(\xi, \eta, \zeta)$.

The acoustic integral includes the Fourier transform of the second-order velocity auto-covariance function $R^{v}(\underline{\mathrm{y}}, \underline{\eta}, \tau)=\overline{v_{x}(\underline{\mathrm{y}}, t) v_{x}(\underline{\mathrm{y}}+\eta, t+\tau)} . R^{v}(\underline{\mathrm{y}}, \underline{\eta}, \tau)$ is modelled in accordance with the analytical Gaussian/exponential shape function suggested by Tam and Auriault [8].

$$
R^{v}(\underline{y,} \eta, \tau)=R^{v}(\underline{y,} 0,0) \exp \left[-|\tau| / \tau_{s}\right] \exp \left[-\left(\xi-\bar{u}_{c} \tau\right)^{2} / l^{2}\right] \exp \left[-\left(\eta^{2}+\zeta^{2}\right)^{2} / l_{\perp}^{2}\right]
$$

which allows the Fourier transform inside the acoustic integral to be computed analytically, and the final expression for the far-field sound pressure spectral density of the Morris and Miller model [6] is given by

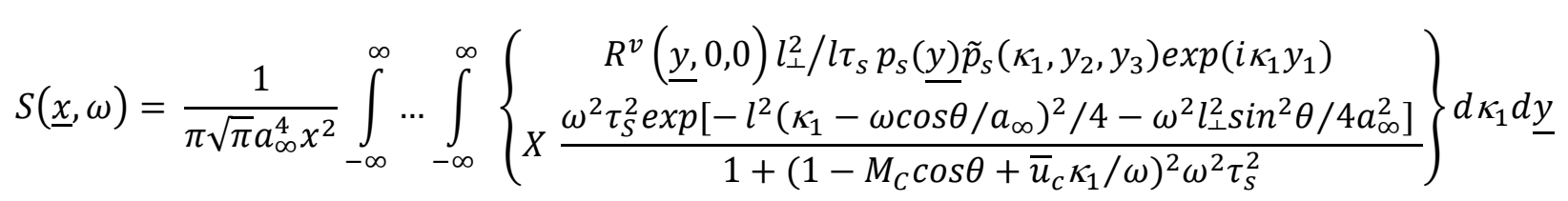

where the correlation scales $\tau_{s}, l_{\perp}$ and $l$ need further modelling since the latter are not available from RANS calculations, which are routinely used for design optimisation studies. It is the modelling of these correlation scales that is the main focus of the current paper.

Let us consider two normally distributed random processes, $X(t)$ and $Y(t)$ which correspond to two noise generation processes in the jet that contribute to the same total velocity fluctuations contributing to far-field noise. Clearly, the two processes are correlated as they are related to the same physical process of BBSAN generation. Let us assume that process $X(t)$ corresponds to dissipation of turbulent eddies and $Y(t)$ corresponds to scattering of the same turbulent eddies by the shock cells. Referring to the experimental 
results of Tan et al. [18], the first process can be related to the random part of the total turbulent velocity fluctuations and the second process can be referred to the correlated part of the same. Let us denote the correlation time scales (variances) of the two processes as $\tau_{s 1}$ and $\tau_{s 2}$, respectively. The variance of the process responsible for turbulent dissipation is expected to be proportional to the turbulent kinetic energy dissipation rate $\tau_{s 1} \sim k / \varepsilon$. The variance of the process responsible for the turbulent eddy scattering by the shock cells is assumed to be inversely proportional to the axial mean-flow velocity gradient $\tau_{s 2} \sim\left(\frac{\partial U_{1}}{\partial y_{1}}\right)^{-1}$. The proportionality coefficients in both cases can be regarded as calibration parameters of the model and will be determined later. In accordance with the mathematical statistics [21], the variance of the sum of two random processes can be expressed through their individual variances and their covariance so that

$$
\tau_{s}=\tau_{X+Y}=\sqrt{\tau_{s 1}^{2}+\tau_{s 2}^{2}+\operatorname{cov}\left(X\left(\tau_{s 1}\right), Y\left(\tau_{s 2}\right)\right)}
$$

where, the covariance function $\operatorname{cov}\left(X\left(\tau_{s 1}\right), Y\left(\tau_{s 2}\right)\right)$ needs to be specified to close the statistical model.

For example, let us assume that the standard deviation of process $Y(t)$ is zero. That is, there is no part of the total turbulent velocity fluctuation that is related to the turbulent eddy scattering by the organised shock-cell structure and only the random part of the velocity fluctuations responsible for diffusion is retained. This leads to the acoustic correlation time scale identical to the standard Morris and Miller model [6] that stipulates $\tau_{s} \sim k / \varepsilon$.

To retain both parts of the BBSAN process, the two-process model (5) can be firstly re-arranged to the following form [22]

$$
\tau_{s}=\tau_{s 1} \cdot f\left(\tau_{s 1}, \tau_{s 2}\right)
$$

where the function $f\left(\tau_{s 1}, \tau_{s 2}\right)=\sqrt{1+\left(\tau_{s 2} / \tau_{s 1}\right)^{2}+\operatorname{cov}\left(X\left(\tau_{s 1}\right), Y\left(\tau_{s 2}\right) / \tau_{s 1}^{2}\right)}$ can be modelled in accordance with the following dimensional analysis and physical considerations:

- the only dimensionless combination involving the two time scales $\tau_{s 1}$ and $\tau_{s 2}$ is $\tau_{s 2} / \tau_{s 1}$, 
- function $f\left(\tau_{s 1}, \tau_{s 2}\right)$ is expected to grow with the strength of the shock cells, $\left|\frac{\partial U_{1}}{\partial y_{1}}\right| \sim \tau_{s 2} / \tau_{s 1}$; the growth corresponds to the initial shear layer region, which is typically associated with highfrequency jet noise and this is where the shock cells are particularly strong,

- for small shock cell strengths, e.g. $\left|\frac{\partial U_{1}}{\partial y_{1}}\right| \ll f_{a}$, where $f_{a}$ is a characteristic acoustic frequency typical of BBSAN, function $f\left(\tau_{s 1}, \tau_{s 2}\right)$ should tend to 1 so that the correlation scale is fully determined by turbulent dissipation,

- function $f\left(\tau_{s 1}, \tau_{s 2}\right)$ cannot be smaller than 1 since the total noise cannot be smaller than the noise produced by the random component of the total velocity fluctuations.

Thus, to the leading order, $f\left(\tau_{s 1}, \tau_{s 2}\right)$ can be approximated by a power law function truncated by the minimum value at $f\left(\tau_{s 1}, \tau_{s 2}\right)=1$ :

$$
\begin{gathered}
f\left(\tau_{s 1}, \tau_{s 2}\right)=\left(\frac{\tau_{s 1}}{\tau_{s 2}}\right)^{\alpha} \quad \text { if } \frac{\tau_{s 1}}{\tau_{s 2}}=\frac{k(\boldsymbol{y})}{\varepsilon(\boldsymbol{y})} \cdot\left|\frac{\partial U_{1}}{\partial y_{1}}\right|>1 \\
f\left(\tau_{s 1}, \tau_{s 2}\right)=1, \text { else }
\end{gathered}
$$

where $\alpha=$ const $\geq 0$, is a new model calibration parameter which needs to be determined in a similar way that the proportionality coefficient of the correlation time scale $\tau_{s 1} \sim k / \varepsilon$ needs to be determined for the original Morris and Miller model [6]. For example, the calibration can be achieved by matching the farfield spectra predictions of the model with the far-field measurements at $90^{\circ}$ polar angle for one jet condition.

In the following sections 2.1 and 2.2, details of several frequency dependent models which use the turbulent energy dissipation scale similar to the original Morris and Miller model [6], as well as those of the new mixed scale model, are provided. All of these models have been implemented and their comparison for BBSAN modelling will be discussed in the rest of the paper. 


\subsection{Frequency Dependence of Turbulence Scales}

Table 1 summarises details of the three models of frequency dependent scales used in the current study in the framework of BBSAN modelling.

Table 1 Time and Length Scale Models

\begin{tabular}{|c|c|c|c|}
\hline Model & Time- Scale & Length Scale & $\begin{array}{l}\text { Calibration } \\
\text { Constants }\end{array}$ \\
\hline Page et al. [18] & $\begin{array}{l}\tau_{s}(\boldsymbol{y}, \boldsymbol{S t})=c_{\tau} \frac{2 \pi}{\omega_{c}+\omega} \\
\omega_{c}(\boldsymbol{y})=c_{\omega} \frac{2 \pi U_{1}(\boldsymbol{y})}{l}\end{array}$ & $\begin{array}{l}l(\boldsymbol{y})=k(\boldsymbol{y})^{3 / 2} / \varepsilon(\boldsymbol{y}) \\
l_{\|}(\boldsymbol{y})=c_{l} l(\boldsymbol{y})\end{array}$ & $\begin{array}{l}c_{\tau}=4.33 \\
c_{\omega}=6.23, \\
c_{l}=0.18\end{array}$ \\
\hline Morris and Boluriaan [19] & $\tau_{s}(\boldsymbol{y}, \boldsymbol{S t})=\frac{l_{x}}{k^{1 / 2}}$ & $l_{x}(\boldsymbol{y}, \boldsymbol{S t})=c_{l} \frac{D_{j} s(\omega)}{S t}$ & $\begin{array}{l}c_{l}=2.0 \\
c_{s} / 2 \pi=3.58\end{array}$ \\
\hline & & $\begin{array}{l}s(\omega)=1-\exp \left(-\frac{c_{S} S t l_{s}(\boldsymbol{y})}{D_{j}}\right) \\
l_{s}(\boldsymbol{y})=k(\boldsymbol{y})^{3 / 2} / \varepsilon(\boldsymbol{y})\end{array}$ & \\
\hline Miller [12] & $\tau_{s}(\boldsymbol{y})=c_{\tau m i x} \frac{k(\boldsymbol{y})}{\varepsilon(\boldsymbol{y})}$ & $\begin{array}{l}l_{x}(\boldsymbol{y})=c_{\text {lmix }} \frac{k(\boldsymbol{y})^{3 / 2}}{\varepsilon(\boldsymbol{y})} \\
l_{x}(\boldsymbol{y}, \boldsymbol{S t})=l_{x}(\boldsymbol{y}) \frac{1-\exp \left(-c_{f} S t\right)}{c_{f} S t}\end{array}$ & $\begin{array}{l}c_{\text {tmix }}=0.06, \\
u_{\text {smix }}=1.00 \\
c_{f}=11.25, \\
c_{\text {lmix }}=0.40 .\end{array}$ \\
\hline
\end{tabular}

\subsection{Mixed Scale Model}

The mixed scale model is the one developed in the present study that combines the mixing noise-type turbulent energy dissipation time scale $k(\boldsymbol{y}) / \varepsilon(\boldsymbol{y})$ from Morris and Miller [6] and the time scale that characterises the interaction of the turbulent eddies with the shock cells, which can be scaled with the axial mean-flow velocity gradient $\left(\frac{\partial U_{1}(y)}{\partial x}\right)^{-1}$. The acoustic time scale is defined in accordance with $(8,9)$. The space scale is related to the time scale in accordance with the relation suggested by Frendi et. al [20]:

$$
l(\boldsymbol{y})=c_{l m i x} \sqrt{k(\boldsymbol{y})} \tau_{s}(\boldsymbol{y})
$$


The cross-stream length scale is the same as the one used in the baseline model by Morris and Miller [6] using the relation $l_{\perp}=c_{\perp} l$. The constants have the values as: $\alpha=2.0 ; c_{\tau}=5.25 ; c_{l}=1.25$ and $c_{\perp}=$ 0.25. Following Kalyan and Karabasov [9], these parameters have been obtained by matching the model predictions with respect to the far-field sound spectra data from the experiment for one jet condition and one observer angle and then keeping the model calibration parameters constant for all other jet cases and observer angles.

A quantitative comparison of the above correlation scale models, the effective noise sources based on these scales, and the far-field sound predictions based on these sources will be provided in section 4 that is devoted to axisymmetric jet noise modelling.

\section{Implementation}

The implementation of the model involves two phases - gathering the near field properties of the jet followed by the calculation of the acoustic spectra. Figure 2 describes the implementation listing out the predominant steps involved.

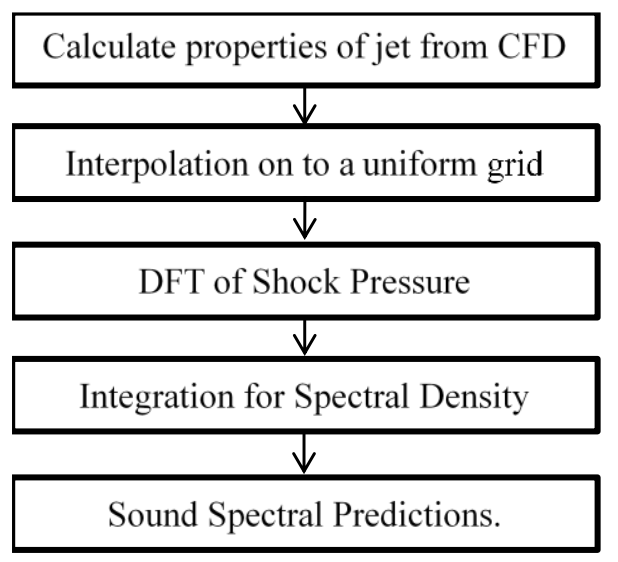

Fig. 2 Flow diagram of model implementation [9]

\subsection{Flow-Field Modelling}

For the purpose of validation, the convergent-divergent and convergent nozzle cases considered in Morris and Miller [23] and Miller et al. [24] have been used. The flow-fields have been computed using a RANS $k-\omega$ SST model, due to its stability with adverse pressure gradients and separating flow. The present 
simulations make use of the second-order Roe scheme for spatial discretization. The boundary conditions include an inlet boundary condition at the inflow region of the nozzle, free-stream boundary conditions at the far-field domains, and a downstream pressure condition for the jet outflow.

A symmetry boundary condition along the symmetry plane has been applied. The insides of the nozzles have slip walls near the inlet boundary of the nozzle, which transform into no-slip walls just ahead of the converging section of the nozzles. In the far-field, the boundary conditions are fixed by setting an ambient pressure and temperature, and specifying a very small free-stream velocity. The free-stream Mach was set at 0.001 for stability of the boundary conditions in the RANS calculation.

For the 3D simulations, the computational domain in the $(x, y, z)$ basis of Cartesian coordinates, where $x$ is aligned with the jet axis had the following dimensions ( $x$ times $y$ times $z$ ) $40 D \times 10 D \times 10 D$. The total number of grid elements used was about 3,000,000 and the typical number of iterations for the cases presented was 90,000 . The grid is specified such that close to the nozzle exit, the corresponding distance from the first grid point to the wall is found by setting the $y+$ distance to 1 . The areas of interest are primarily in the jet plume and the shear layer of the jet and hence these regions are more refined in all the cases in comparison with the interior of the nozzle and the freestream regions. The grid refinement has been accompanied by running several RANS calculations sequentially to verify that the target viscous wall grid resolution has been achieved as required. Refinement of the grid also proved to be essential to capture the shock cells as accurately as possible, as they form the crucial input to the BBSAN model. RANS solutions are iterated until convergence is achieved. Typically, the iterations are stopped when the corresponding convergence residuals of the RANS equations drop down to the round-off error level $\left(\sim 10^{-13}\right)$ or the residuals of the various equations being solved stagnate at a level of three or more orders of magnitude less than the initial residuals.

The operating conditions of the two jet cases computed are summarized in Table 2.

Table 2 Operating and Design Conditions

\begin{tabular}{lllllllll}
\hline \hline Nozzle & $\mathbf{M}_{\mathbf{d}}$ & $\mathbf{M}_{\mathbf{j}}$ & $\mathbf{N P R}$ & $\mathbf{T T R}$ & $\mathbf{D}(\mathbf{m})$ & $\mathbf{D}_{\mathbf{j}}(\mathbf{m})$ & $\mathbf{u}_{\mathbf{j}}$ & $\mathbf{f}_{\mathbf{c}}$ \\
Convergent-Divergent & 1.50 & 1.30 & 2.77 & 1.0 & 0.0127 & 0.01209 & 385.7 & 31897 \\
Convergent & 1.00 & 1.50 & 3.67 & 1.0 & 0.0127 & 0.01377 & 427.5 & 31040 \\
\hline \hline
\end{tabular}




\subsection{Acoustic Modelling}

The methodology for the acoustic modelling follows the steps as listed out in fig. 2 . The files containing the flow-field data are read and the integration of the model equation is done on structured grids of uniform spacing between each grid point. Interpolation is done on two regions using an inverse-distance weighted method, which will hereafter be referred to as the integration domain and the wavenumber domain. Both the domains have the same locations with respect to the locations in the $r$ or $y$, and the $z$ directions. The integration region encloses the potential core of the jet and extends up to $11 D$ in the axial direction and $1 D$ in the $r$ or $y$, and the $z$ directions. The variables that are interpolated on this region are all the properties that define the jet, viz. $p, k, \Omega$ and the velocity components. The specific dissipation rate, $\Omega$ is converted into viscous dissipation rate, $\varepsilon$ using $\varepsilon=0.09 k \Omega$, where, $k$ is the turbulent kinetic energy as mentioned by Wilcox [25]. The wavenumber domain almost extends twice the length of the potential core in the axial direction and the range is determined by the minimum distance between the points in the axial direction. The wavenumber spacing is given as $2(\pi / L)$, where $L$ is the length of the domain in the stream-wise direction. The fluctuations of the shock pressure, $p_{s}$ are obtained by subtracting the ambient pressure, $p_{\infty}$ from the static pressure, $p$. After the application of a Hanning window, the Discrete Fourier Transform (DFT) of shock pressure is calculated. A correction for the Hanning window is applied by multiplying the resultant with a factor of $8 / 3$. More details on the flow data post-processing can be found from Kalyan and Karabasov [9].

For the Morris and Miller model [6], the correlation scale models are given as $\tau_{s}=c_{\tau} k / \varepsilon ; l=c_{l} k^{3 / 2} / \varepsilon$ and $l_{\perp}=c_{\perp} l$, where the calibration parameters are $c_{\tau}=1.25, c_{l}=3.50$ and $c_{\perp}=0.30$ for the baseline calculations. These are compared with the other three frequency dependent scales listed in Table 1 and the mixed scale model that was discussed in Section 2. In all cases, the noise spectra (Power Spectra Density) are computed based on the standard reference pressure, $p_{r e f}=2.0 \times 10^{-5} \mathrm{~Pa}$,

$$
S P L=20 \log _{10}\left(\frac{\sqrt{S(\underline{\mathrm{x}}, \omega)}}{p_{\text {ref }}}\right)+10 \log _{10}\left(U_{j} / D_{j}\right)
$$


Losses associated with atmospheric humidity and absorption are not considered. The experimental data against which the predictions have been validated have humidity and atmospheric absorption corrections applied so that the comparisons are all lossless.

\section{Axisymmetric jet modelling}

\subsection{Flow-Field Results}

The accuracy in obtaining solutions for the flow-field data is important because they provide the foundation for the acoustic prediction model used. The solutions of the RANS calculations obtained are compared with those obtained numerically and experimentally in [23, 24]. The flow data available for comparison in this study is limited to the jet regions between $x / D=0$ to 2 , where, $x / D=0$ corresponds to the nozzle exit. While a more complete flow solution validation campaign will be the subject of further work, the first several diameters of jet flow tend to determine the acoustic jet behaviour at high frequencies that is of primary concern here.

These plots are presented in figs. 3 and 4 for the over-expanded and the under-expanded axisymmetric jet cases. Only comparisons for the total pressures and Mach are presented in this section. Profiles of static pressure comparisons can be found in Kalyan and Karabasov [9]. The flow-field data is corrected to yield profiles of local stagnation pressure at a point that would hypothetically be located behind a Pitot probe to account for the normal shock effects that occur due to the presence of the Pitot probe in supersonic flow. The total pressure after the shock and the static pressure before the shock are related to the Mach number ahead of the shock using the Rayleigh-Pitot equation [26].

$$
p_{02}=p_{1}\left[\frac{(\gamma+1) M_{1}^{2}}{2}\right]^{\frac{\gamma}{\gamma-1}}\left[\frac{(\gamma+1)}{2 \gamma M_{1}^{2}-(\gamma-1)}\right]^{\frac{1}{\gamma-1}}
$$

where, $p_{02}$ is the total pressure after the shock, and $p_{l}$ and $M_{l}$ are the static pressure and Mach number before the shock respectively. 
The total pressure profiles demonstrate excellent agreement, while the Mach profiles demonstrate reasonable agreement with the reference experimental data and computational solutions digitised from Morris and Miller [23] and Miller et al. [24]. The minor differences could because of a slight difference in the shock cell spacing due to the difference in the solvers that have been used. The discrepancies with the experiments is caused by the interaction of the oblique shock waves and the probes inside the flow. For the Mach profiles, the spreading rates, indicated by the lower gradients of pressure have been captured reasonably well for both cases.

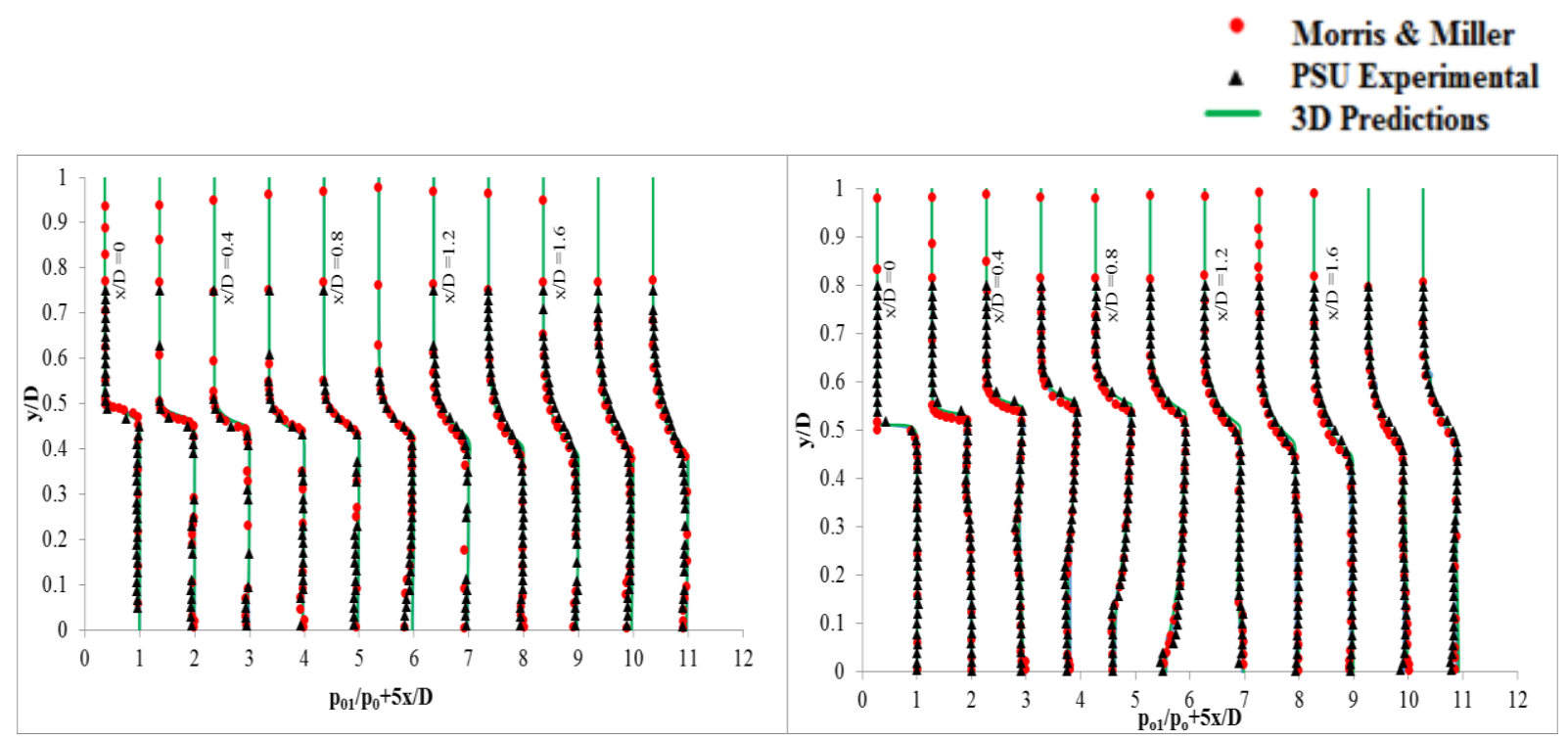

(a)

(b)

Fig. 3 Non-dimensionalized total pressure for (a) CD nozzle and (b) convergent nozzle [9].

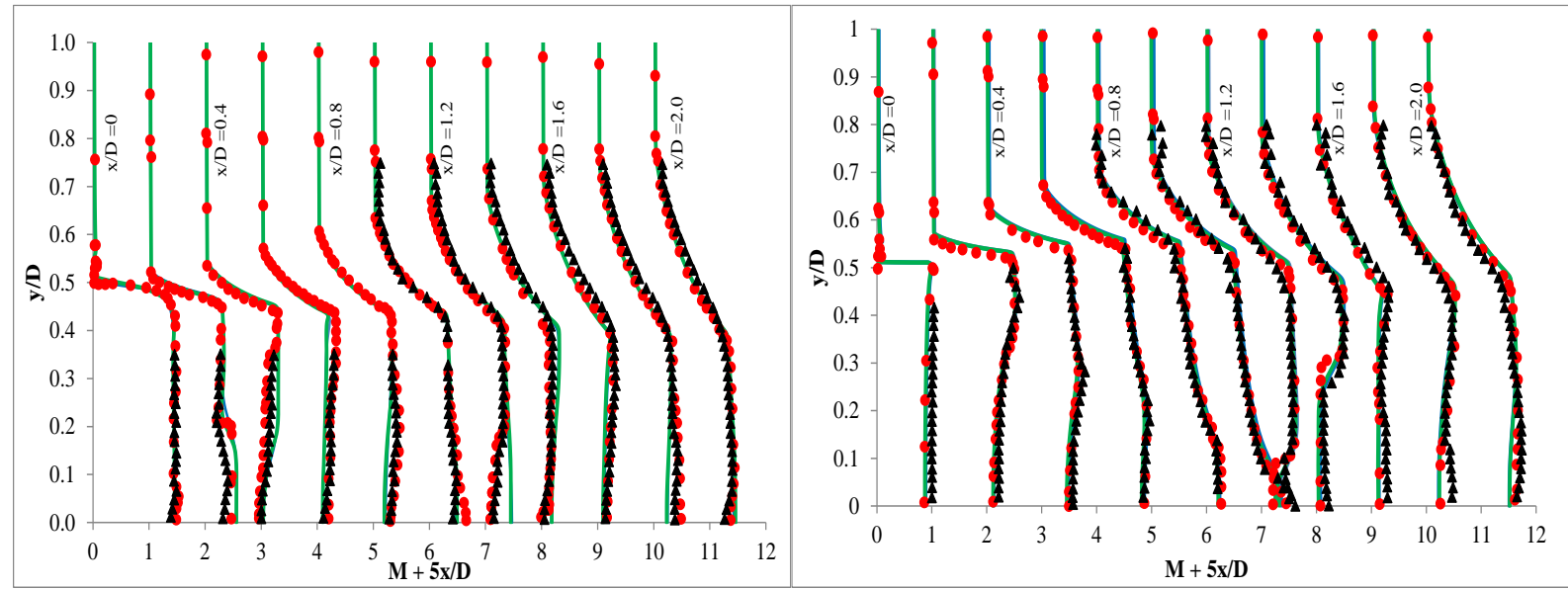

(a)

(b)

Fig. 4 Non-dimensionalized Mach profiles for (a) CD nozzle and (b) Convergent nozzle [9] 
Further validation details for the static pressure profiles in the same jet flow region can be obtained in Kalyan and Karabasov [9].

\subsection{Acoustic Modelling Results}

For the two baseline cases of the axisymmetric convergent-divergent and convergent nozzles, which correspond to the over-expanded and the under-expanded jet cases, respectively, several modifications of Morris and Miller model [6] are considered as well as the implementation of the original model by the authors. The modifications are based on three frequency-dependent scale models and the new mixed scale model which were discussed in section 2. For each of the axisymmetric nozzles, fig. 5 shows the axial correlation length scale distribution as predicted by the five models under consideration.

The distributions correspond to the nozzle lip-line, centreline, and the inner boundary of the shear layer. The latter location is approximated by a straight line joining the nozzle lip to the end of potential core where the shear layers merge together. This is the location typical of peak BBSAN sources where the shock cells are strong while the velocity fluctuations remain highly turbulent. For the axial length scale of the Morris and Boluriaan model [14], which is the only frequency-dependent spatial scale out of the five models, the results are plotted for $\mathrm{St}_{\mathrm{D}}=2$. For most jet locations, this correlation scale distribution is within $5 \%$ variation as compared to the same model predictions for $\mathrm{St}_{\mathrm{D}}=1$, which corresponds to the characteristic peak BBSAN frequency. Besides, $\mathrm{St}_{\mathrm{D}}=2$ is a typical high-frequency Strouhal number at which the original Morris and Miller model [6] is known to lead to significant under predictions.

The comparison of the correlation scale solutions shows that most frequency-dependent correlation scales from the literature converge to the result of the frequency-independent-scale Morris and Miller model [6] for all cases. The frequency-dependent Miller model [16] predicts a similar distribution shape but under predicts the absolute value of correlation scale by $20-30 \%$ as compared to most of the models. For the lipline and the inner shear layer boundary, the correlation scales of the new mixed scale model are not only amplified in comparison to the other models but also show a much more uneven behaviour with several prominent spikes. 
(a)

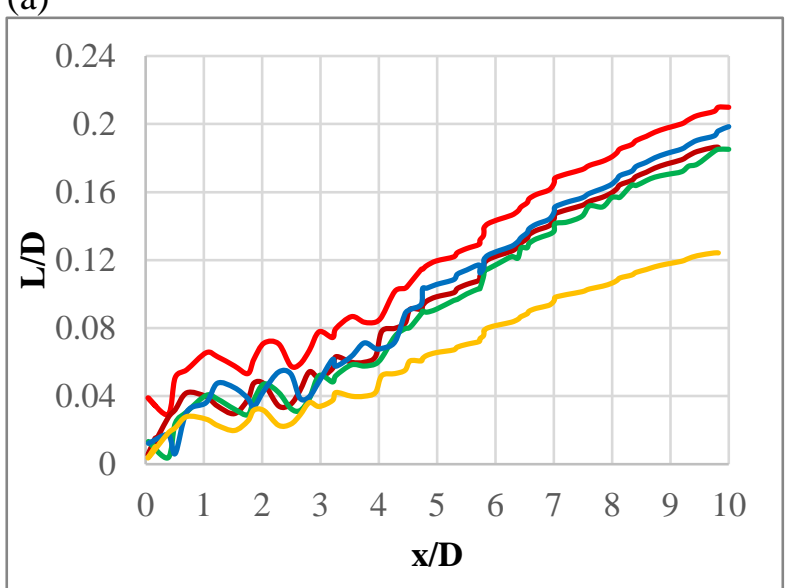

(b)

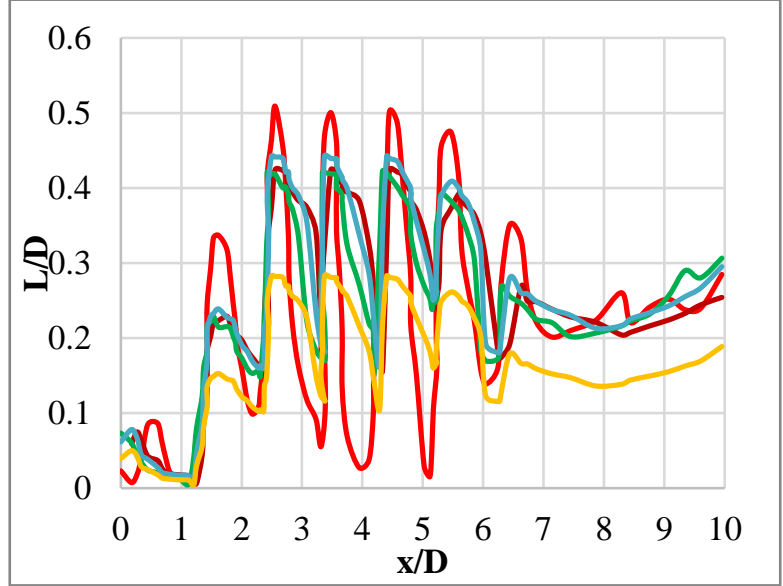

(c)

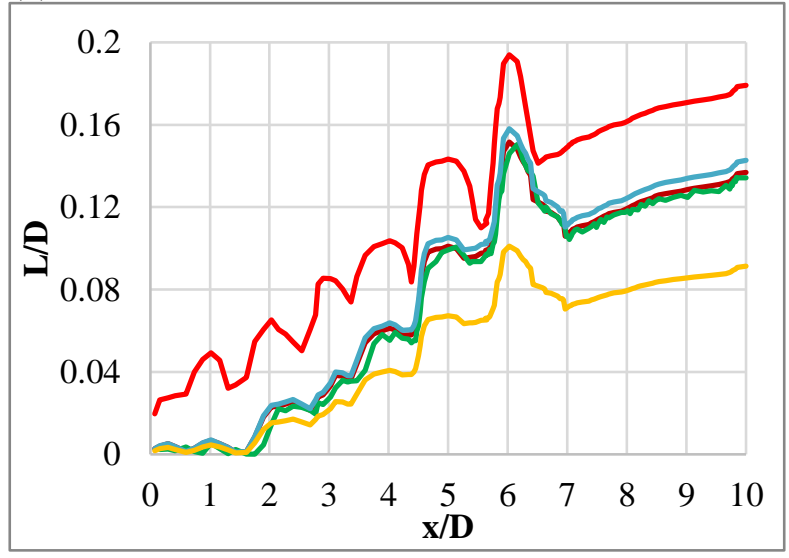

(d)

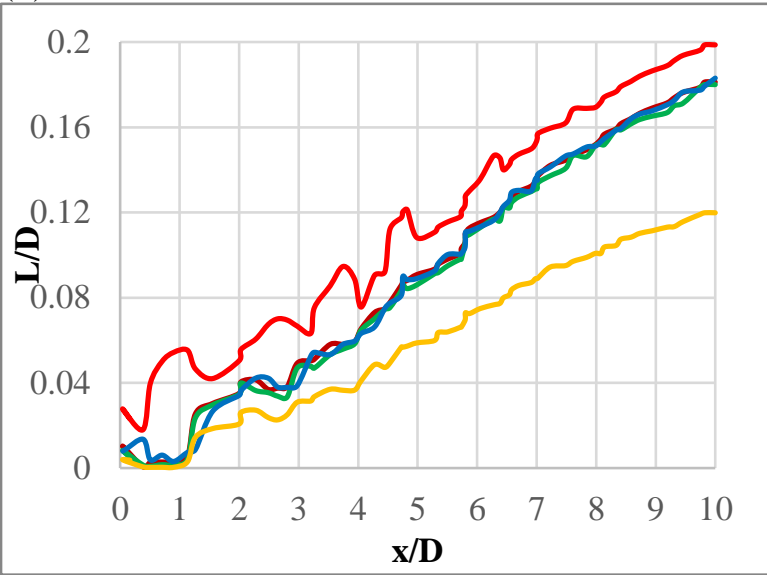

(e)

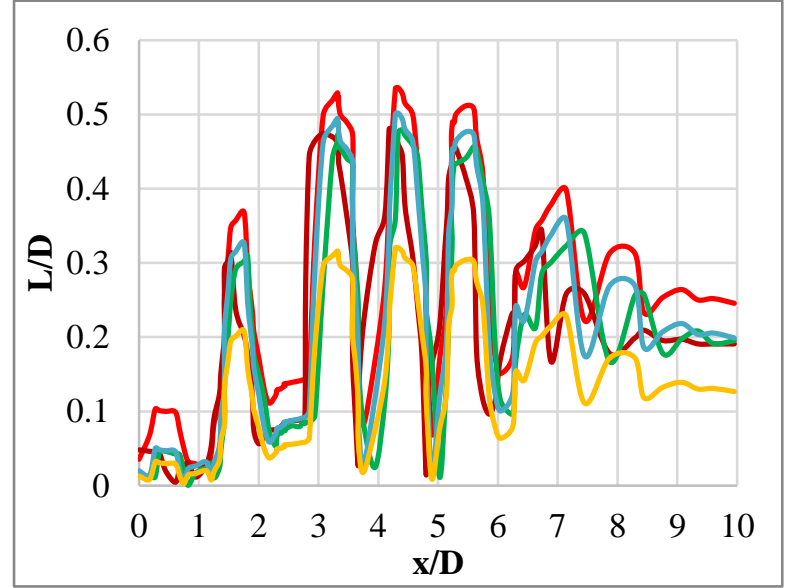

(f)

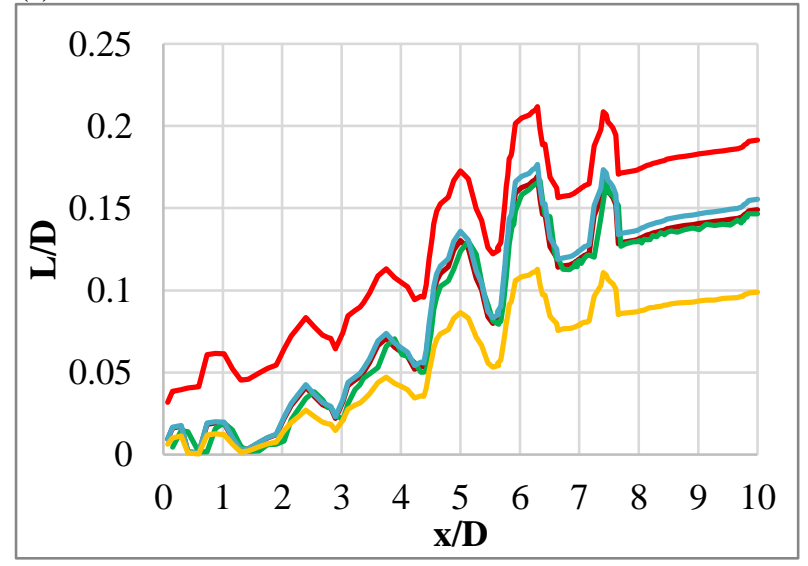

Fig. 5 Length scale comparisons for CD nozzle along (a) lip-line, (b) centreline, (c) inner shear layer boundary and for convergent nozzle along (d) lip-line, (e) centreline, (f) inner shear layer boundary. 
The difference between the models is especially notable for the convergent nozzle. The difference between the mixed scale model and the rest of the models is most pronounced over the first several jet diameters. The latter locations can be expected to be particularly important for high-frequency noise generation.

For a qualitative comparison with the experimental data available, the model scale distributions can be compared with the integral correlation scales obtained from the PIV measurements for a similar convergent jet case considered by Tan et al. [18] (fig.1). First of all, all models agree with the experiment that there are distinct peaks located along the regions of the shock reflection points. Along the centreline, all peaks are located along the regions of the shock cell centre. The correlation scale grows in the shear layer and oscillates around some small value along the centreline relative to the oscillations. All models, except for the frequency-dependent Miller model [16] which shows some scale under prediction as noted earlier, predict similar maximum levels of correlation scale values over the first 10 jet diameters. In dimensionless units based on the nozzle diameter, the maximum dimensionless scale values are about 0.6 for the centreline location and 0.16-0.2 for the shear layer location, which are in good agreement with the experimental data of Tan et al. [18], for the correlation scales based on the total velocity fluctuations.

For the lip-line and the inner shear layer locations over the first 2-3 jet diameters, there are some notable qualitative differences between the correlation scale distributions considered. The correlation scale distribution corresponding to the mixed scale model resembles the shear-layer distribution of the correlation scale from Tan et al. [18], which is based on the total velocity fluctuations. Indeed, both the model and the experiment show a very uneven behaviour with some prominent peaks and both start from some nonnegligible value of the scale, $\mathrm{L} / \mathrm{D}=0.02-0.03$ close to the nozzle exit. In contrast to this, all other models show a more linear distribution along the axial jet coordinate that starts from a virtually zero scale value at the nozzle exit. For example, the smooth behaviour of the correlation scale in case of the CD nozzle is more consistent with the experimental correlation scale obtained from the random part of velocity fluctuations rather than from the total velocity fluctuations in the analysis of Tan et al. [18]. 

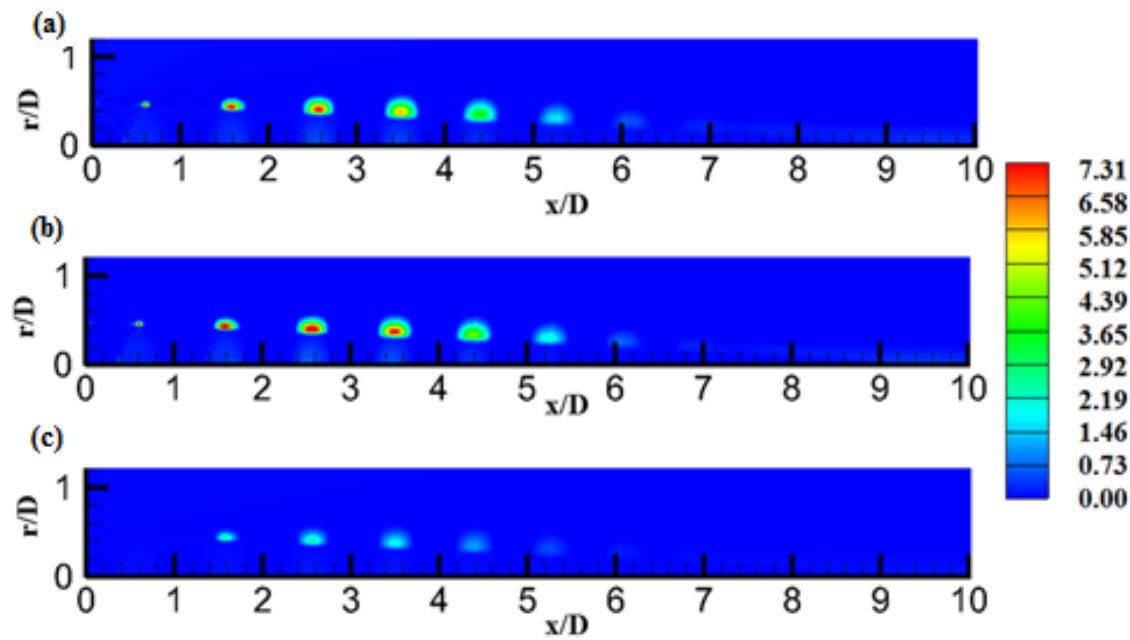

(d)

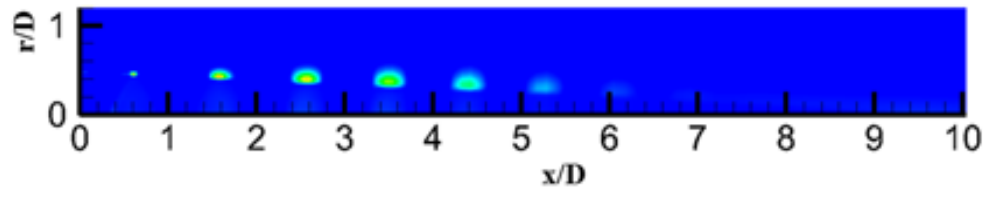

Fig. 6 Spatial distribution of acoustic source density for CD nozzle using Morris and Miller model

[5] at (a) $\mathrm{St}_{\mathrm{D}}=1$ and (c) at $\mathrm{St}_{\mathrm{D}}=2$; mixed scale model at (b) at $\mathrm{St}_{\mathrm{D}}=1$ and (d) at $\mathrm{St}_{\mathrm{D}}=2$.

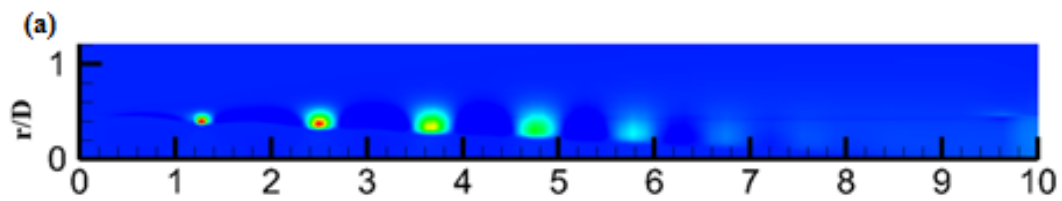

(b)
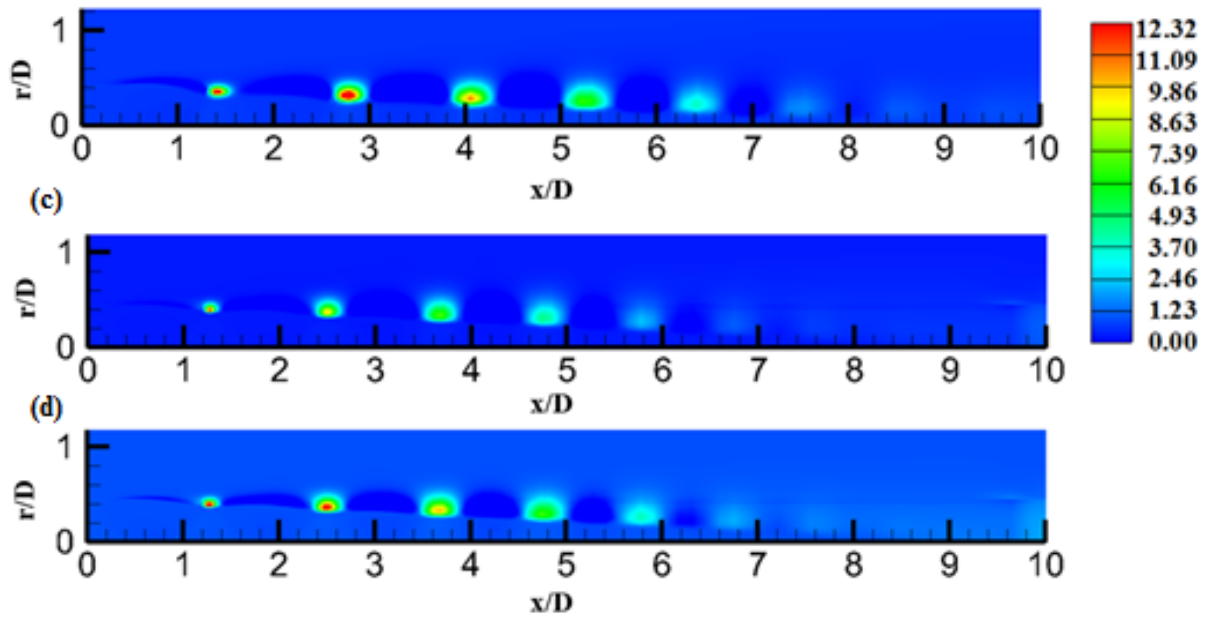

Fig. 7 Spatial distribution of acoustic source density for convergent nozzle using Morris and Miller model [5] at (a) $\mathrm{St}_{\mathrm{D}}=1$ and (c) $\mathrm{St}_{\mathrm{D}}=2$; mixed scale model at (b) $\mathrm{St}_{\mathrm{D}}=1$ and (d) $\mathrm{St}_{\mathrm{D}}=2$. 
To further investigate the differences between the standard Morris and Miller model [6] and its new implementation based on the mixed scale model, the effective source density in cylindrical-polar coordinates $(x, r)$ corresponding to the integrand function of (6) has been integrated around the circumferential direction $r d \theta$.

The source density corresponds to a representative observer angle, $90^{\circ}$ to the jet flow and two characteristic frequencies. One frequency corresponds to $S t_{D}=1$ that is close to the expected BBSAN peak noise frequency and the other corresponds to a twice higher frequency, $S t_{D}=2$, where the sound spectra predictions of the standard Morris and Miller model [6] and its improved version based on the mixed scale model are expected to differ. Figures 6 and 7 show the contours of the resultant source density normalized by the reference acoustic pressure $p_{r e f}=2 \times 10^{-5} \mathrm{~Pa}$, and the nozzle exit diameter. In comparison with the standard model, the mixed scale model results in more pronounced source regions associated with shock reflection points. The difference is particularly noticeable for the high-frequency case of the convergentdivergent nozzle.

(a)

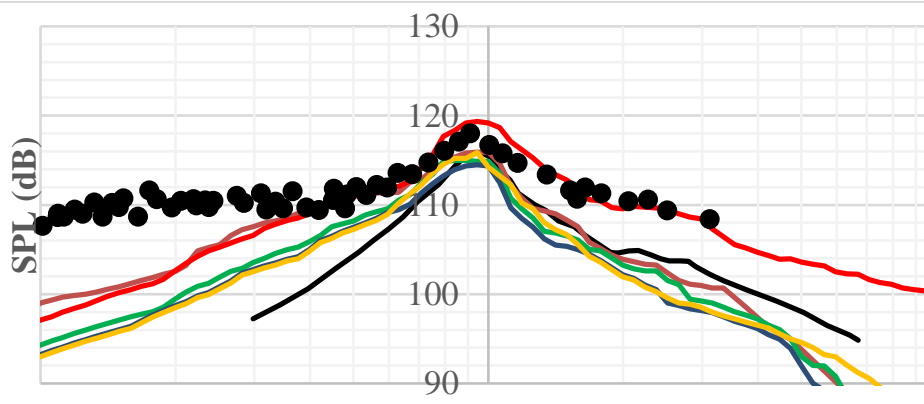

$\stackrel{1}{\mathbf{S t}}$
10

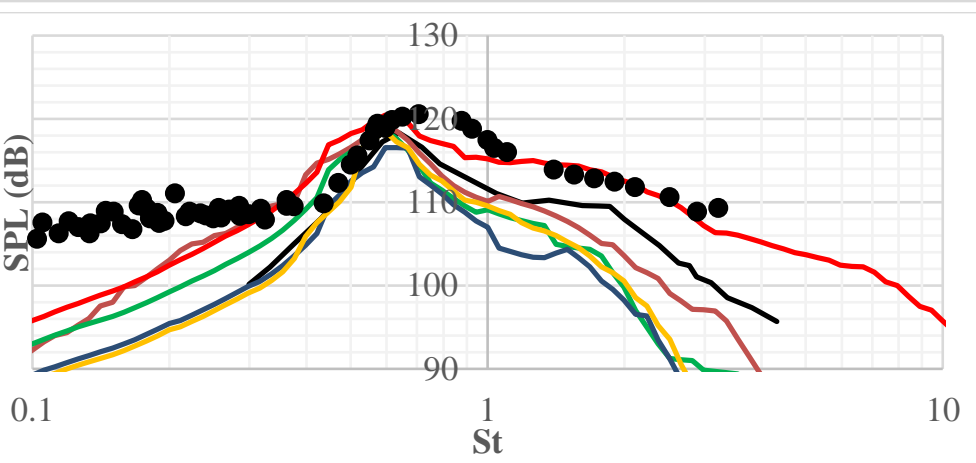

- PSU Experimental Morris \& Miller Implementation Morris \& Miller

- Page et. al Morris \& Boluriaan Implementation - Miller Mixed Scale Model

(b)

Fig. 8 Acoustic spectral comparisons for CD nozzle at (a) $90^{\circ}$ and (b) $120^{\circ}$ [22]. 


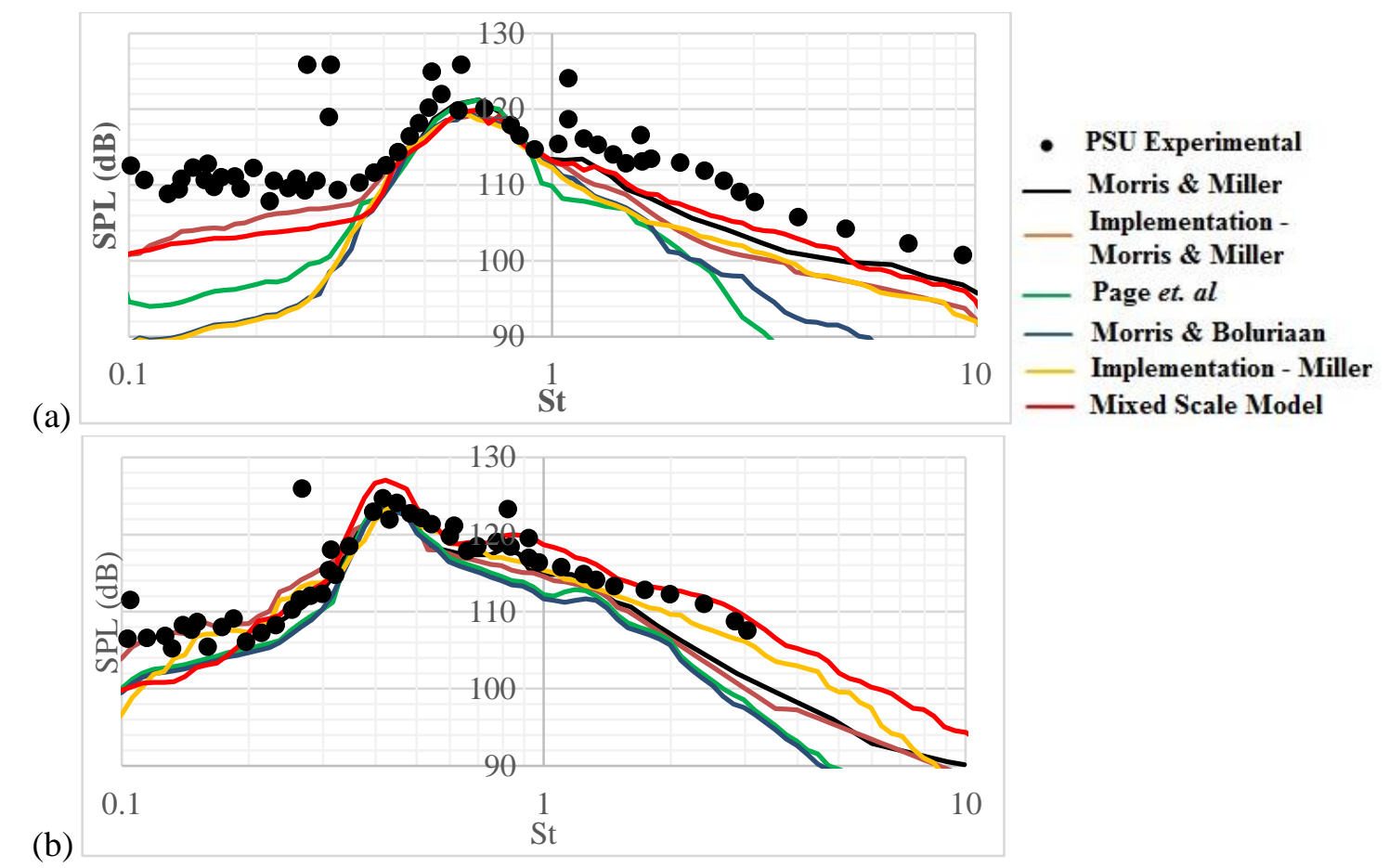

Fig. 9 Acoustic spectral comparisons for convergent nozzle at (a) $90^{\circ}$ and (b) $120^{0}$ [22].

As the final validation step, the far-field noise spectra predictions of all five acoustic models considered together with the digitised noise spectra predictions from the original publication by Morris and Miller [6] are compared with the experimental data available for the same convergent and convergent-divergent jets. Spectral comparison for $90^{\circ}$ and $120^{\circ}$ polar angles with respect to the jet downstream axis and $100 \mathrm{D}$ distance from the nozzle exit are shown in figs. 8 and 9.

All models predict the shift of the noise spectra peak to lower frequencies for a higher angle and capture the peak of the broadband spectra in comparison with the experiment within $2 \mathrm{~dB}$. However, both the original sound predictions from Morris and Miller model [6], the current implementation of their model as well as its modifications to account for frequency dependent source scales fail to capture the higher frequency decay of the spectra of the over-expanded jet.

This is in contrast with the new mixed scale model which captures high-frequency part of the spectra within $2 \mathrm{~dB}$ for the over-expanded jet case and 3-4dB for the under-expanded jet for both $90^{\circ}$ and $120^{\circ}$ observer angle and frequencies up to $S t_{D}=3$ as compared to the experiment. This can be compared with the accuracy of the original Morris and Miller model [6] that is broadly within 5dB from the experiment for 
both the over-expanded and the under-expanded jet case. Other observer angles which have been considered in this work include $80^{\circ}, 100^{\circ}, 110^{\circ}$ and $130^{\circ}$. For the case of the new mixed scale model, the results for the additional angles are shown in section 5. Generally, the results of the spectral comparison between different models and the experiment follow the same trend as the two angles shown in figs 8 and 9: the mixed scale model gives consistently more accurate predictions in comparison with the original Morris and Miller model [6] and its several frequency-dependent scale modifications considered.

One caveat to keep in mind at this point is that it is very challenging to separate BBSAN from jet mixing noise in the experiment for high frequencies, despite the fact that such source separation is possible in some cases as done by Viswanathan [27]. Since some of the experimental data from Morris and Miller [23], which are used in the current validation, could be potentially polluted by jet mixing noise more validation studies for the new mixed scale model are in order.

The improvement in sound predictions of the mixed scale model is further reflected in fig. 10 which shows the Over-All Sound Pressure Levels (OASPL) directivity for both jets where the experimental data have been obtained by numerical integration of the digitized spectra data from [23,24] while excluding the screech-tone related peaks in the sound spectra of the under-expanded jet. The OASPL comparison shows that the predictions of the current mixed scale model are within $1 \mathrm{~dB}$ from the experiment for most angles. This is compared with the results of the original Morris and Miller model [6] and the frequency-dependentscale Miller model [16], the accuracy of which is similar and is within 2-3dB from the experiment. Following Kalyan and Karabasov [9], the sensitivity of the mixed scale model to the calibration parameters have been investigated to confirm only a nominal difference in the spectra within $1 \mathrm{~dB}$ while varying the model parameters $\alpha=2.0 ; c_{\tau}=5.25 ; c_{l}=1.25$ and $c_{\perp}=0.25$ by 10 percent. 


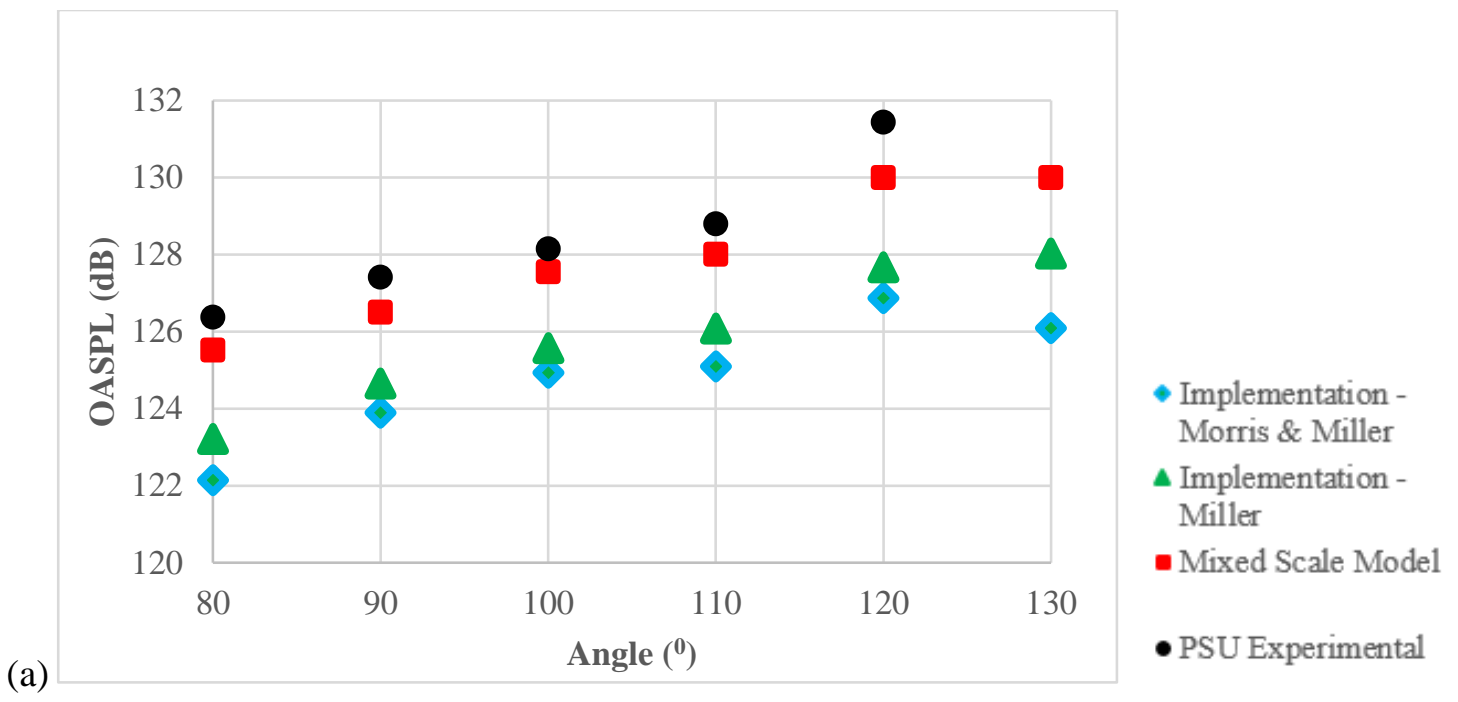

(a)

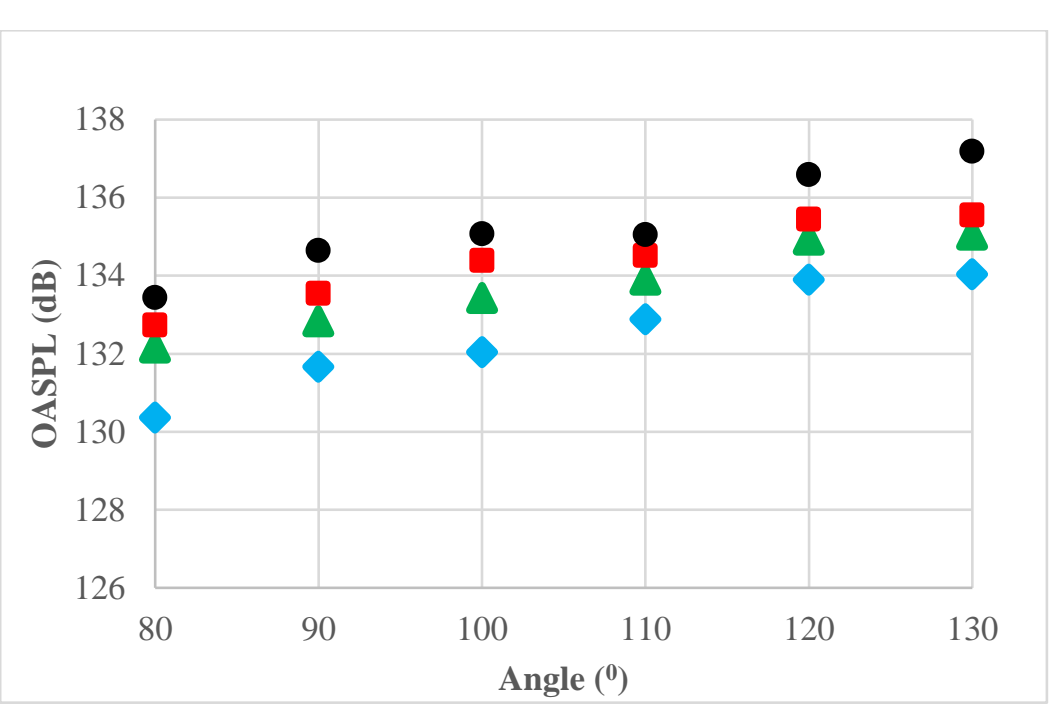

Fig. 10 OASPL directivity comparisons for (a) CD nozzle and (b) convergent nozzle [22].

To conclude this section, it is worth remarking that other examples of fast turn-around-time BBSAN prediction schemes in the literature which can also accurately capture high-frequency noise spectra include those by Tam [28] and Harper-Bourne [10]. The first of these models uses an empirical correction factor to improve the high frequency predictions while the second is fully based on an empirical dataset to achieve the same. These are in contrast with the new mixed scale model that improves high-frequency predictions of the original Morris and Miller model [6] by considering two distinct processes contributing to BBSAN as motivated by the physics. Hence, the latter model is expected to be more robust for design optimisation 
studies. As the first step in this direction, the application of the new mixed scale model for BBSAN modelling of asymmetric jets will be considered in the next section.

\section{Asymmetric Jets}

A way to reduce BBSAN is to diminish the shock cell - turbulence interaction by weakening the strength of the shock cells through breaking the axisymmetry of the jet flow. To study these effects for each baseline case of the axisymmetric convergent-divergent and convergent jets, this section considers several generic elliptic jets of varying eccentricity from Kalyan and Karabasov [9]. The jets correspond to different aspect ratios (AR), viz., 1.5, 2.0 and 3.0 but the same nozzle exit area and jet velocity at the nozzle exit as for the baseline axisymmetric jets so that the thrust is maintained the same. The calculation of the asymmetric jets is performed using the same 3D RANS method, boundary conditions and grid density recommendations, which were found optimal for the axisymmetric jet cases discussed in section 3.

\subsection{Jet Cases}

Figure 11 shows the geometry of the baseline axisymmetric nozzles, which have been modified so that the exit plane of the nozzle assumes an elliptical shape, with as small alterations to the contours of the original nozzle as possible. To maintain the same aerodynamic performance as the baseline axisymmetric nozzle cases, the areas at the exit are maintained the same, while only changing the aspect ratio. Thus, the equivalent axisymmetric diameter, $D_{e q}=0.0127 \mathrm{~m}$ remains the same for all jet cases. 


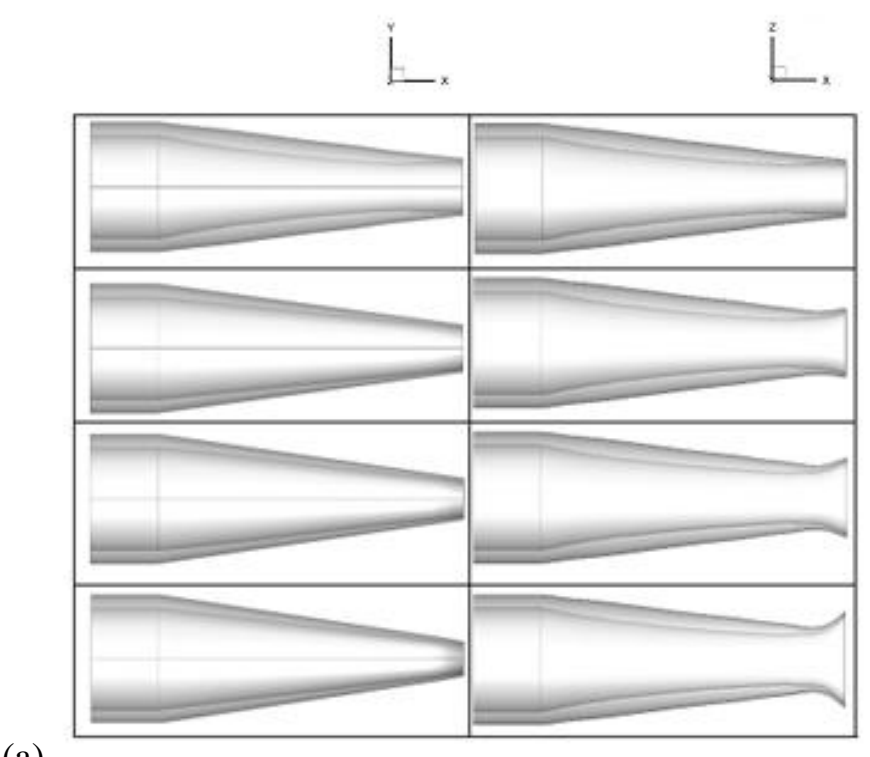

(a)

(b)

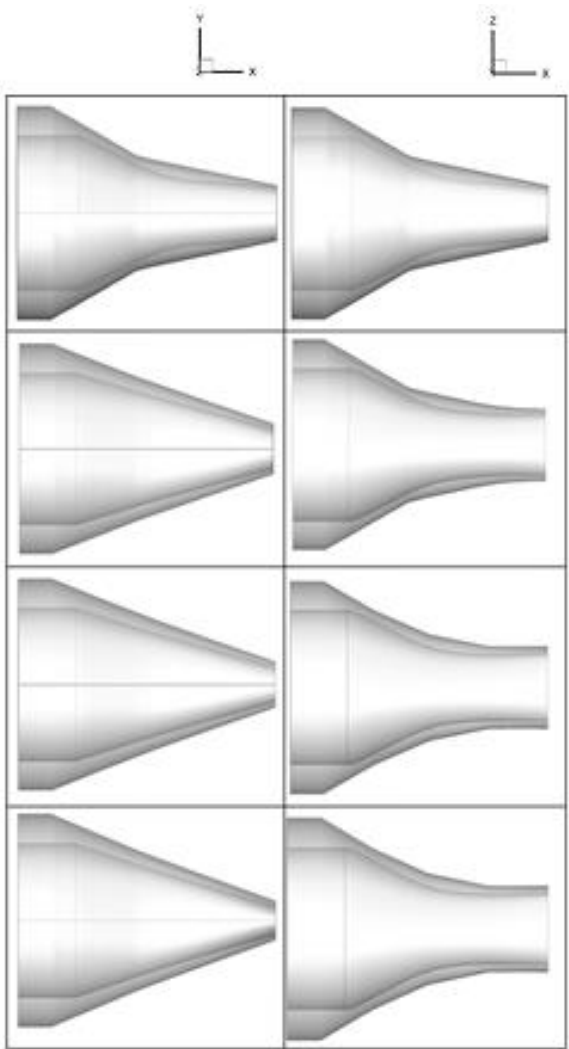

Fig. 11 Nozzle geometries along minor and major axis planes for (a) CD nozzles and (b) convergent nozzles; the aspect ratio increases from $A R=1$ at the top to $A R=3$ at the bottom.

For simplicity of the geometry generation, while modifying the axisymmetric convergent-divergent nozzle geometry, the nozzle profile has been chosen to be convergent-divergent along the major axis plane, whereas, convergent along the minor axis plane. The axisymmetric convergent nozzle profiles have been modified to ensure that the nozzle is convergent along both the planes.

\subsection{Flow-Field Results}

Figure 12 shows the centreline comparisons of non-dimensionalized shock pressure for the overexpanded and the under-expanded jet cases. The shock pressure has been non-dimensionalized with $1 / 2\left(\rho_{j} u_{j}^{2}\right)$. A trend that can be observed here is that as the AR increases, the number of the shock cells and their width reduces. This effect is more marked in the case of the over-expanded jet. 

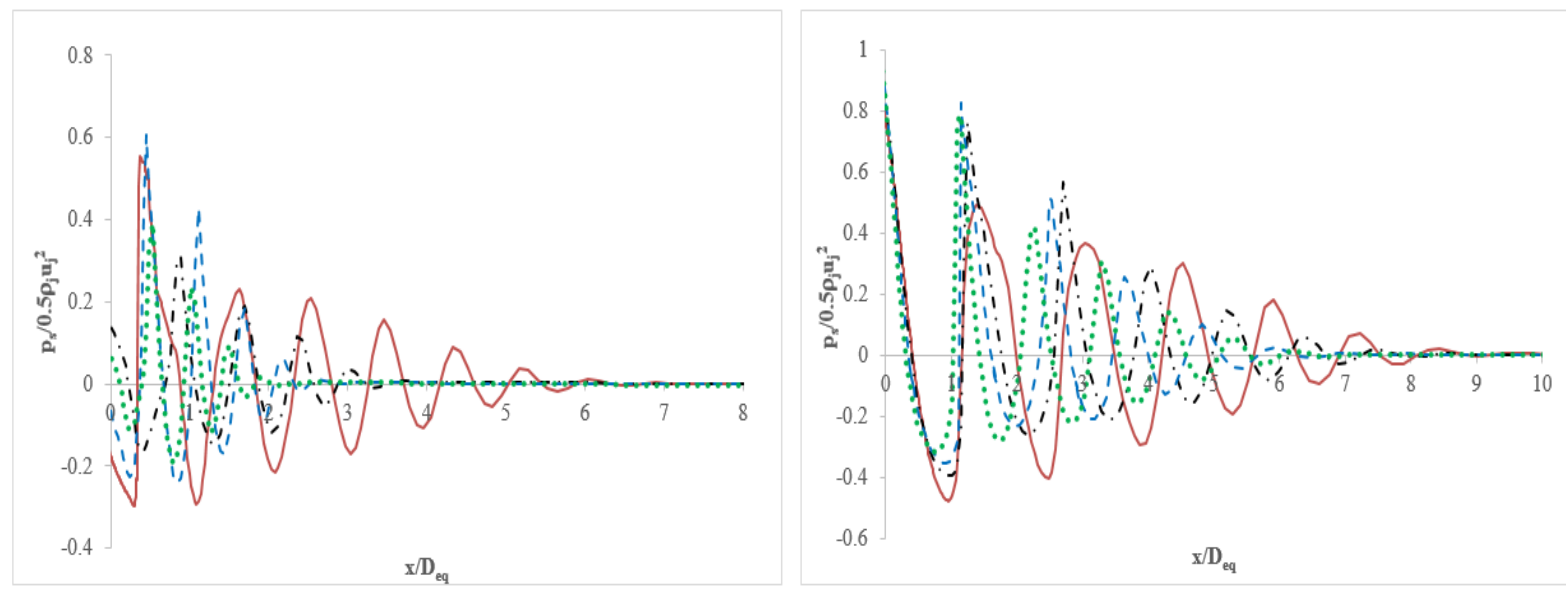

Fig. 12 Non-dimensionalized shock pressure for (a) CD nozzles and (b) convergent nozzles [9].

With just a minimum change in eccentricity, as is in the case of $A R=1.5$, the axial length of the shock cell structure is truncated as much as $50 \%$ in comparison with the reference axisymmetric jet case. For the convergent nozzle, the trend obtained is similar to the one for the convergent-divergent nozzle, although not as pronounced. This difference could be attributed to the way the nozzle profiles, which have been modified from the axisymmetric cases, vary between each other for the different elliptic configurations, as explained above. Comparison of the axial mean-flow velocity profiles between the convergent-divergent and the convergent jets show the same trend as the static pressure profiles. More flow field comparison details can be found in Kalyan and Karabasov [9].

The difference in the development of the turbulent shear layers between the two planes in the elliptic jets considered is further illustrated in fig. 13 which shows the distributions of axial velocity components for the jets at various AR. The shear layers originate from the nozzle lips and their growth acts as a waveguide that contain the shock cells within them. The differences in the structure of the jet potential cores between the major and minor axis planes can be noticed. The shortening of the potential core of the elliptic jets in comparison with the axisymmetric jets shows that the latter jets have a larger zone of high- 
velocity that may be responsible for excessive noise generation in the case of the axisymmetric jets. A detailed investigation of the BBSAN sources in each jet case will be provided at the end of this section.

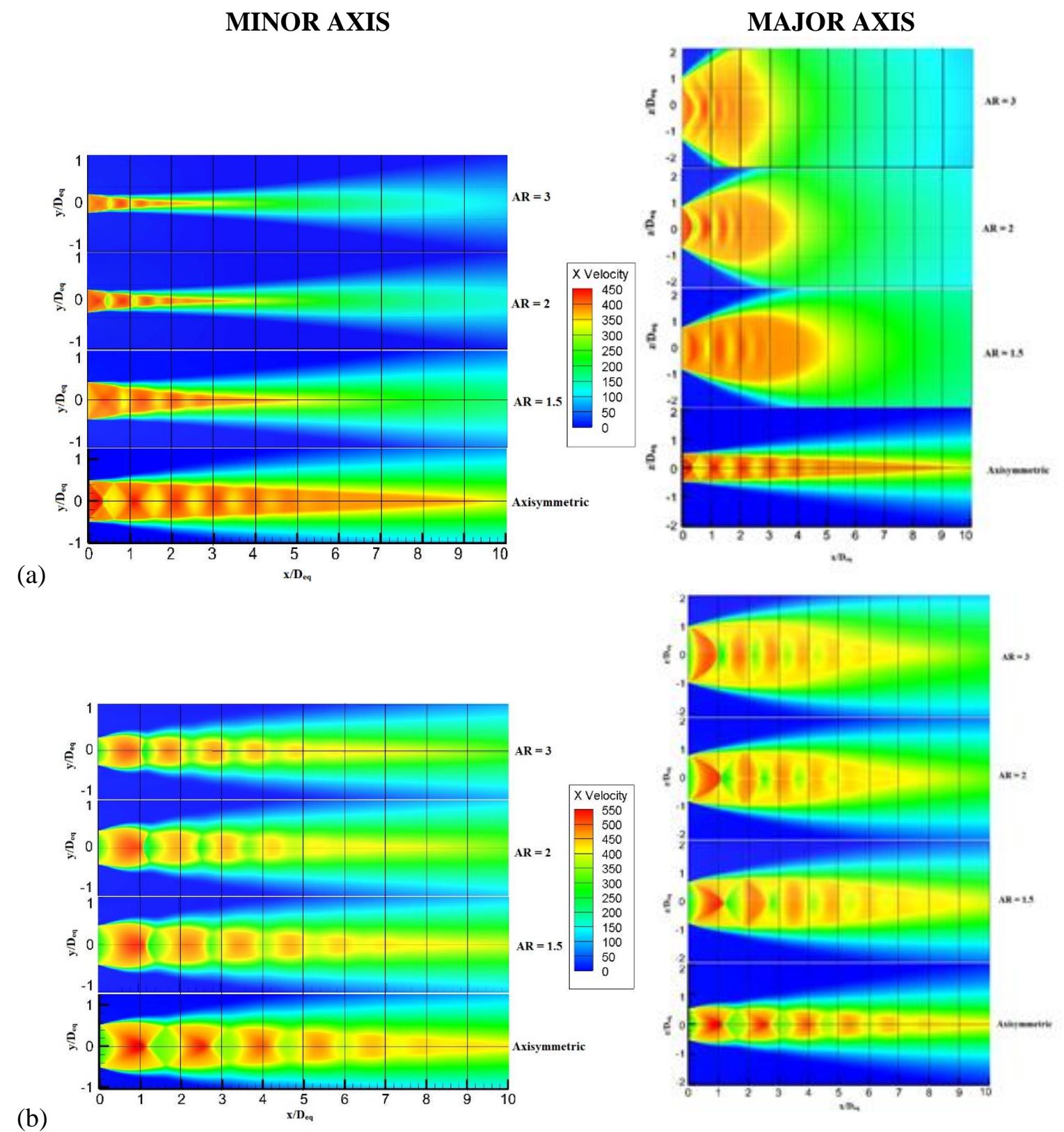

Fig. 13 Axial velocity contours for (a) CD nozzles and (b) convergent nozzles [9].

The contours along the major axis show that the jet spread rates are higher in comparison to the minor axis plane. The values of velocity drop significantly after the shocks. For the over-expanded jet, the axisymmetric potential core extends to almost $10 \mathrm{D}$ while the most elliptic nozzle having $\mathrm{AR}=3.0$ has a potential core that extends only up to $3 D$. An oblique shock originates from the lip of the nozzle and 
terminates into a barrel shock. Prandtl-Meyer expansion waves originate in the second shock cell and form another conical oblique shock, and so on. These barrel shocks become more compressed as aspect ratio increases. This is visible distinctly on the contours along the major axis plane. In case of the under-expanded jet, the reduction in the length of the potential core is not as much as the over-expanded jet and the reduction can be observed only to be up to $20 \%$ of the axisymmetric case, even for the highest $\mathrm{AR}=3.0$. The slightly wavy nature that can be seen in the shear layer region on the contours along the minor axis plane are due to the expansions and shock waves of the shock cell structure. A very small normal shock ahead of the oblique shock can be seen on the contour along the major axis plane. This leads to the creation of a small subsonic region downstream, limited by the slip lines. The slip lines downstream of the barrel shock propagate through the entire shock cell structure. Also, it can be noticed that the conical shock becomes more compressed as AR increases. Figure 14 depicts the same in a more quantitative approach to evaluate the asymmetric shocks by comparing the profiles of dimensionless total pressure on both the major and minor axis planes.

To justify that the dissipation of shock cells was not a consequence of a lack of the grid resolution, which for RANS modelling is typically rather coarse in the flow direction, all the grids have been refined twice the original grid along the axial direction with re-running the calculations. Figures 15 and 16 compare the solutions at two grid resolutions for non-dimensionalized static pressure profiles between $x / D=0$ to $x / D=$ 2 along the major and minor axis for the cases of different aspect ratio CD and convergent nozzles. The difference is nominal and less than $1 \%$ consistently. The grid sensitivity study for Mach and total pressure also showed that the original grid and the refined grid solutions virtually collide. The grid sensitivity investigation thus confirmed that the RANS solutions obtained are reasonably grid independent. 


$$
\begin{aligned}
&--A R=1.5 \\
&-\quad A R=2.0 \\
& \cdots \quad A R=3.0
\end{aligned}
$$

MINOR AXIS

MAJOR AXIS
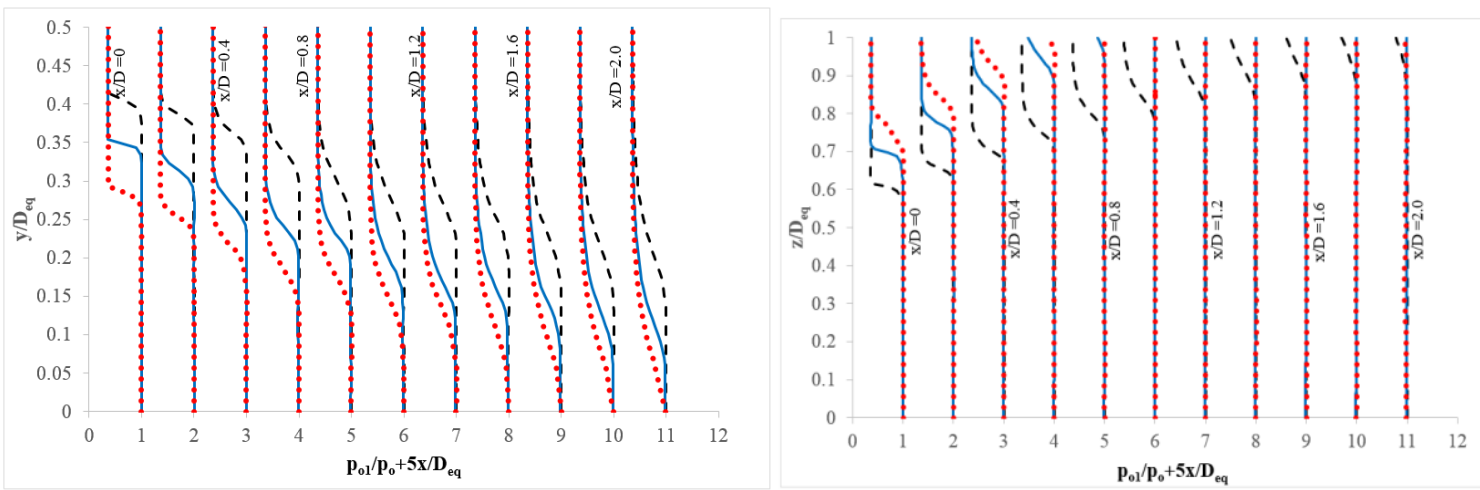

(a)
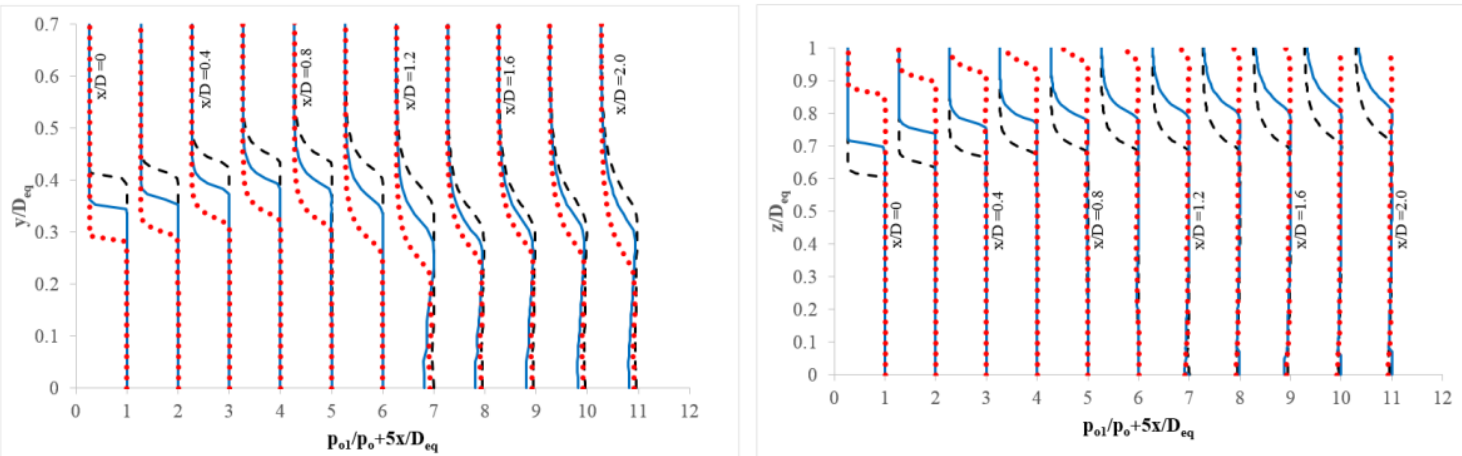

(b)

Fig. 14 Total pressure profiles for (a) CD nozzles, (b) convergent nozzles [9]. 
(b)

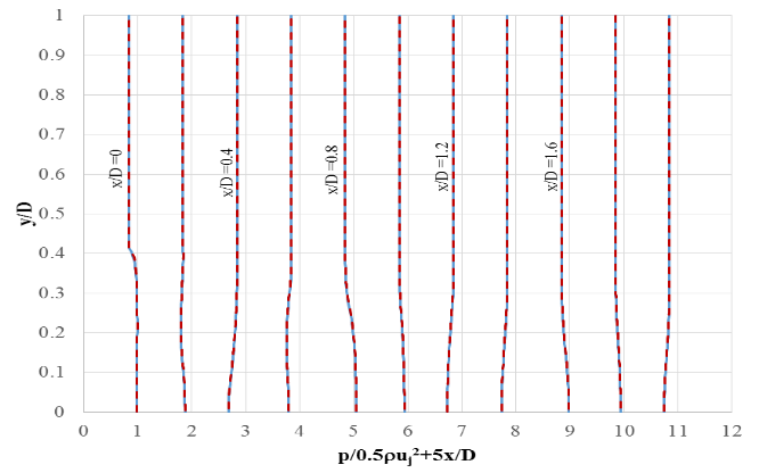

(c)

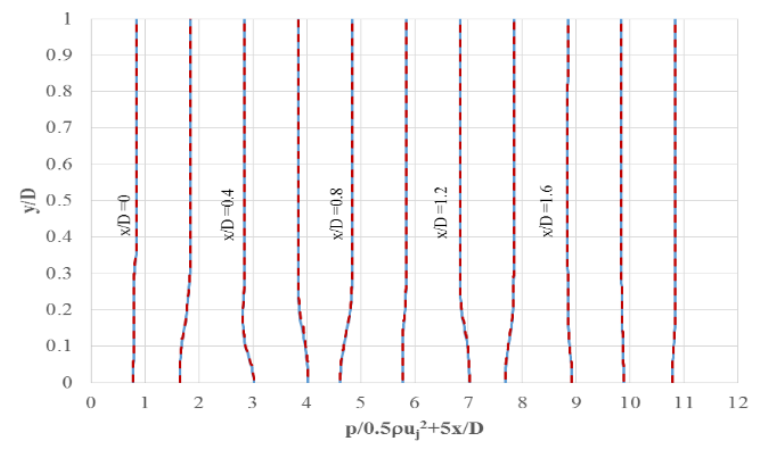

(d)

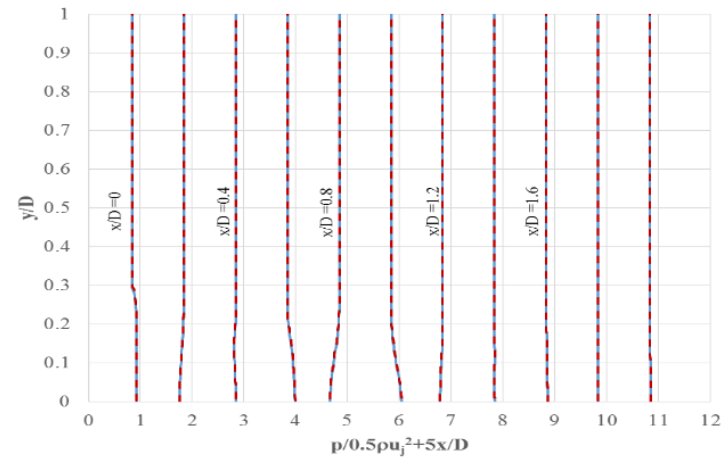

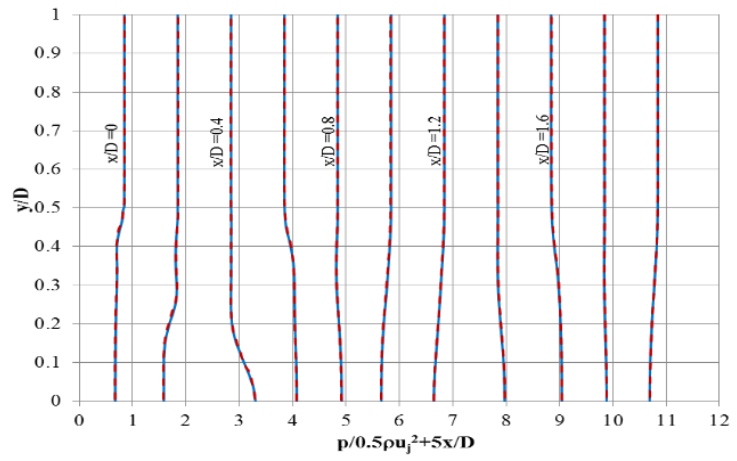

MAJOR AXIS
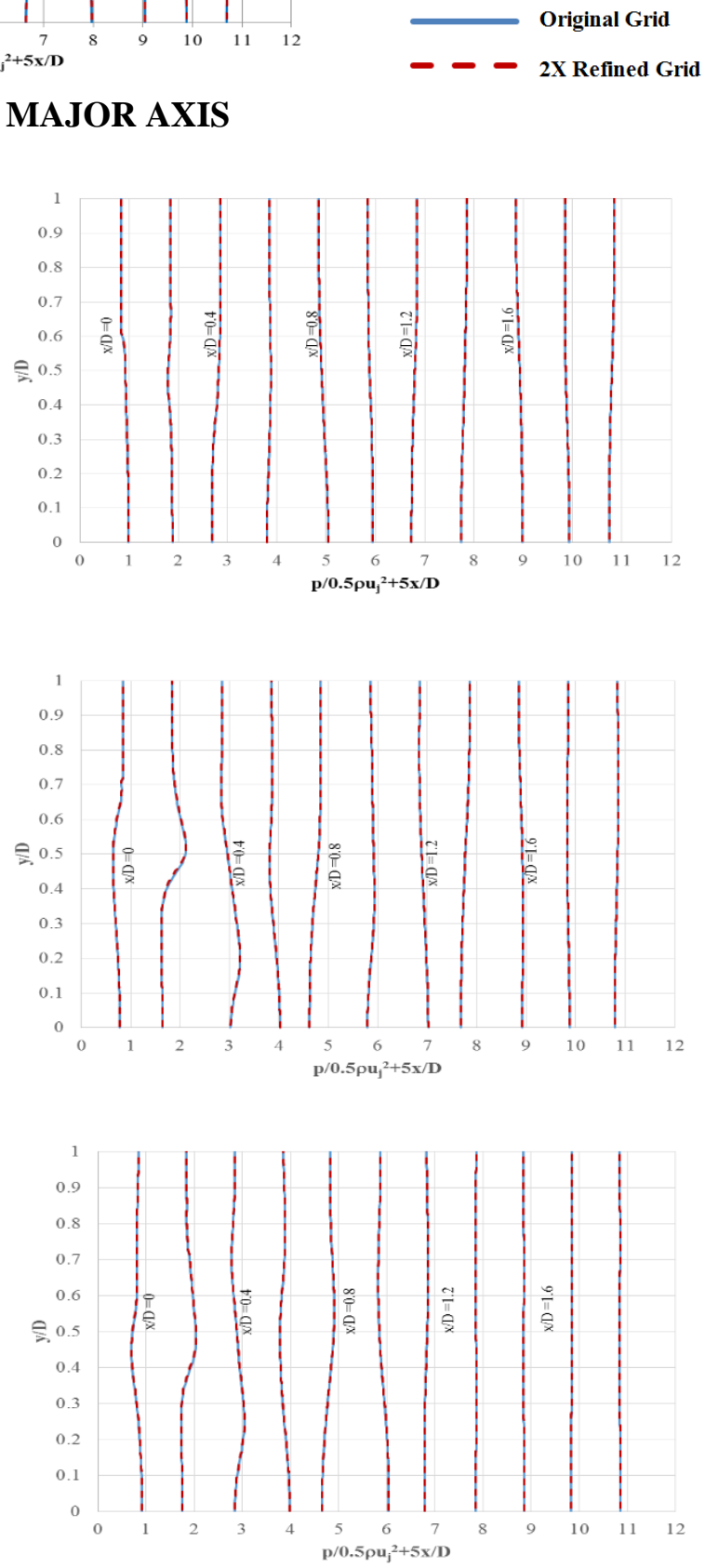

Fig. 15 Static pressure profiles for CD nozzle: (a) AR 1.0, (b) AR 1.5, (c) AR 2.0, and (d) AR 3.0. 


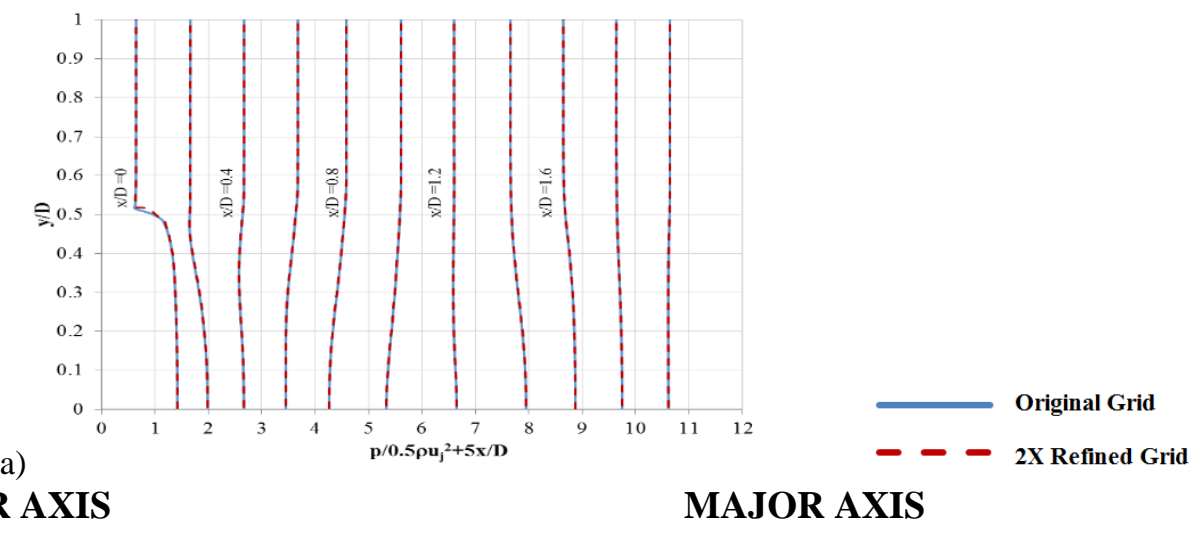

(b)

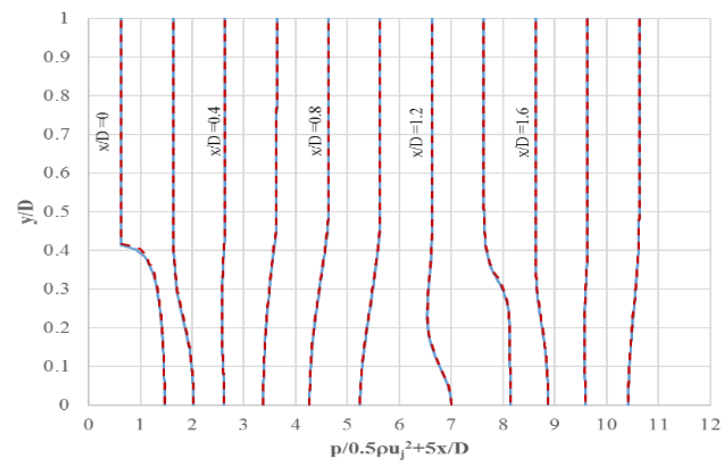

(c)

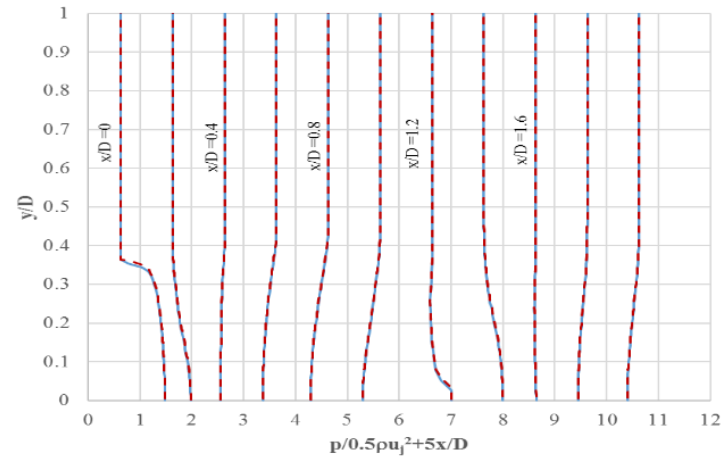

(d)

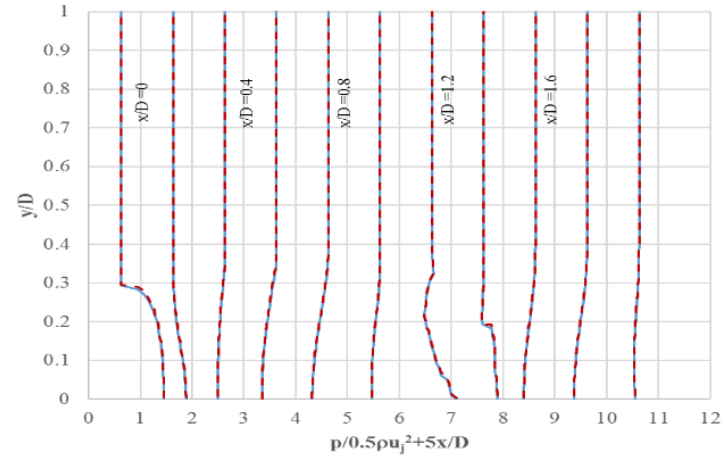

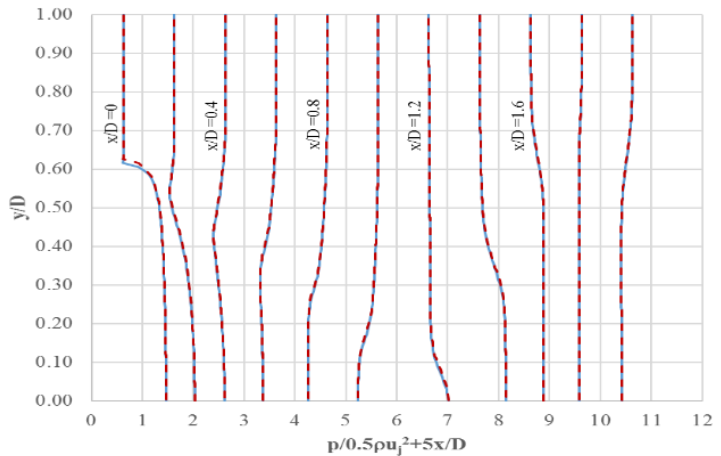
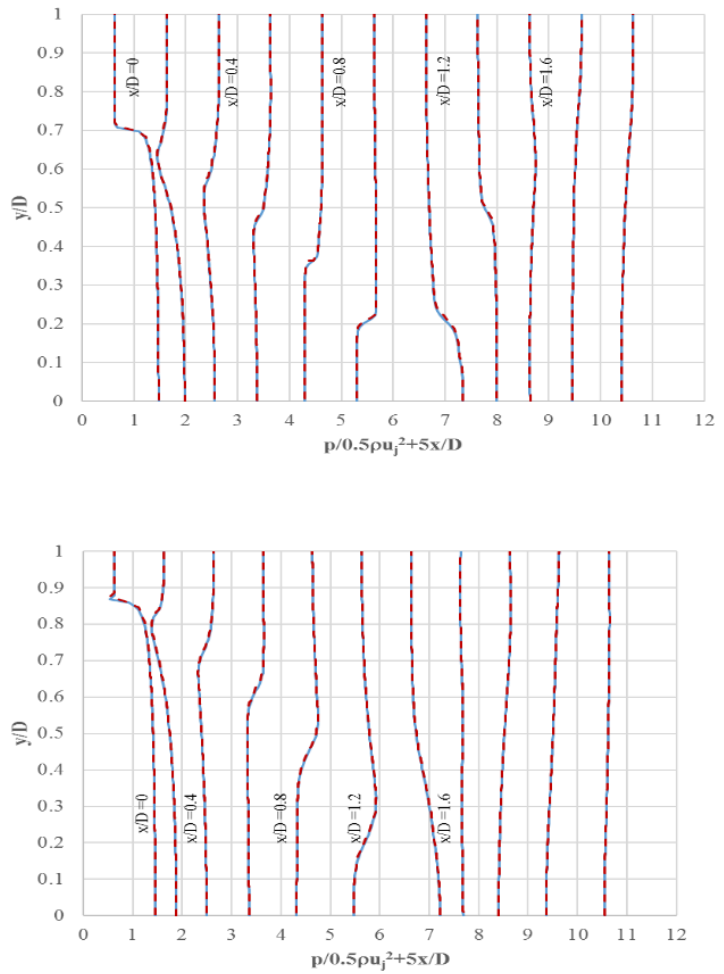

Fig. 16 Static pressure profiles for convergent nozzle: (a) AR 1.0, (b) AR 1.5, (c) AR 2.0, and (d) AR

3.0. 


\subsection{Acoustic Modelling}

The mixed scale model from the previous section is now applied for BBSAN modelling of the asymmetric jets. In section 4, it has been established that the difference in acoustic correlation scales may play a key role for BBSAN generation of axisymmetric jets. Now, the effect of changing the nozzle geometry from axisymmetric to elliptic on the correlation length scale is investigated. For analysis, the scale distribution along the shear layer inner boundary region is chosen as it approximately corresponds to the peak noise source location where both the shock cell strength and the turbulence intensity levels of the jet are significant. The location of the inner boundary of the shear layer is approximated by the line joining each lip-line point with the end of potential core of each jet as for the axisymmetric jets in section 4 . For the asymmetric jets, however, the radial location of the inner shear layer boundary line depends on the azimuthal angle at each location and so do the length scales corresponding to the effective noise sources around the nozzle circumference.
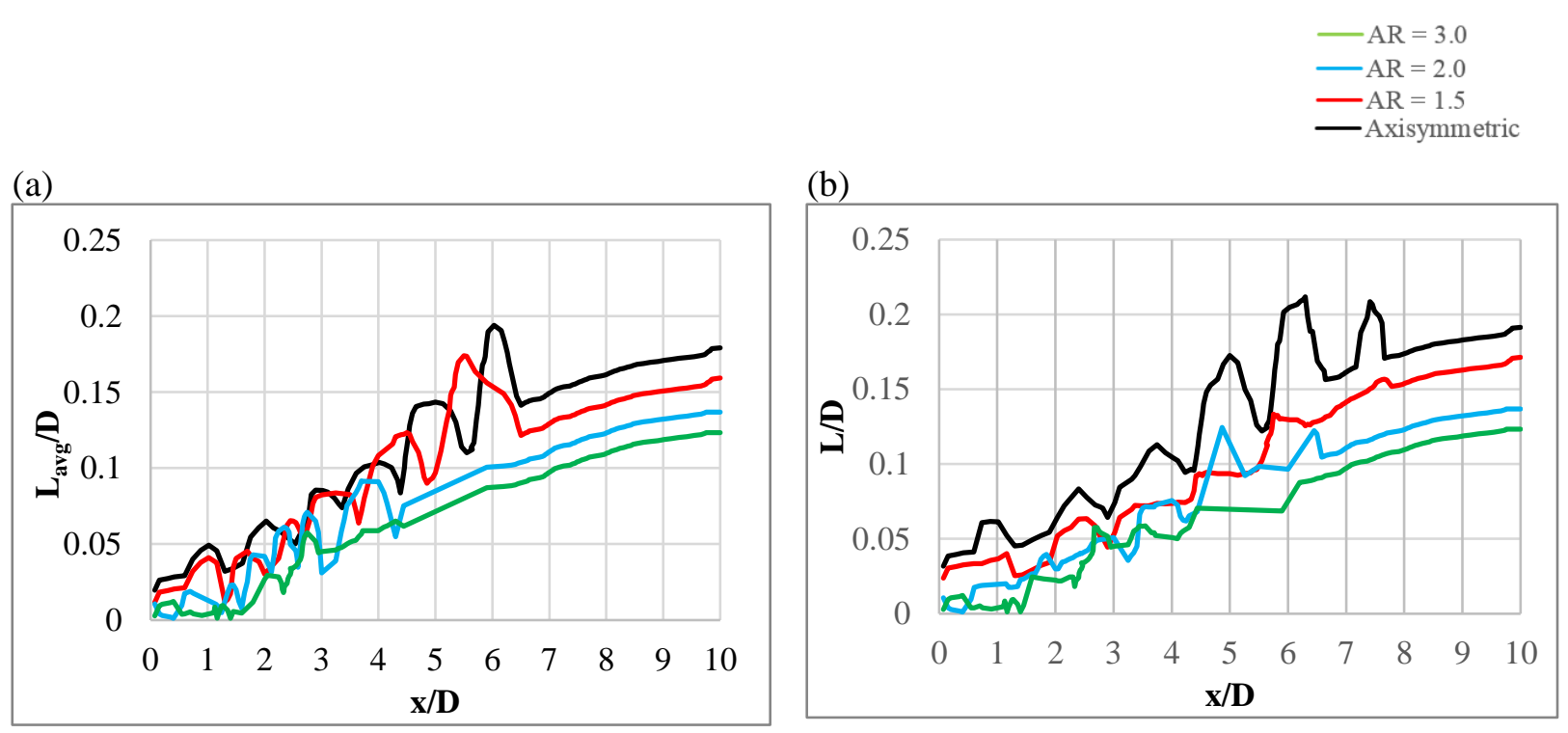

Fig. 17 Length scale distribution comparison of various eccentricity along the inner shear layer boundary corresponding to the peak of effective noise source for (a) CD nozzle; (b) convergent nozzle.

To account for the azimuthal variation of the correlation length scale for elliptic jets of variable AR, the length scale distributions have been averaged circumferentially for each axial jet location and the results are compared with the scale distributions in the reference axisymmetric $\mathrm{CD}$ and convergent jet in fig.17. 
As the aspect ratio increases, the length scales decrease in their magnitude and their distribution becomes smoother and shows a more linear behaviour: the peaks, which correspond to the shock reflection points, become weaker and more closely packed and the initial correlation scale drops down to a small value near the nozzle exit. For the highest aspect ratio jets, $A R=3$, the correlation scale distributions closely resemble the shear layer distribution of the correlation scale based on the random part of the velocity fluctuations from the axisymmetric jet experiment of Tan et al. [18] the shape of which is characteristic of pure jet mixing noise.

To visualise the effect of increasing the elliptic nozzle eccentricity on the effective sound sources, the integrand function of the effective sound source of (6) based on the mixed scale model has been calculated for the peak BBSAN frequency of $\mathrm{St}_{\mathrm{D}}=1$ and then averaged around the circumferential direction to compare the resulting field in $(x, r)$ plane with the sources of the axisymmetric jets from section 4 . Similar to the axisymmetric jet cases, the resulting source density is non-dimensionalized by scaling it by the reference pressure and the equivalent jet diameter.

(a)

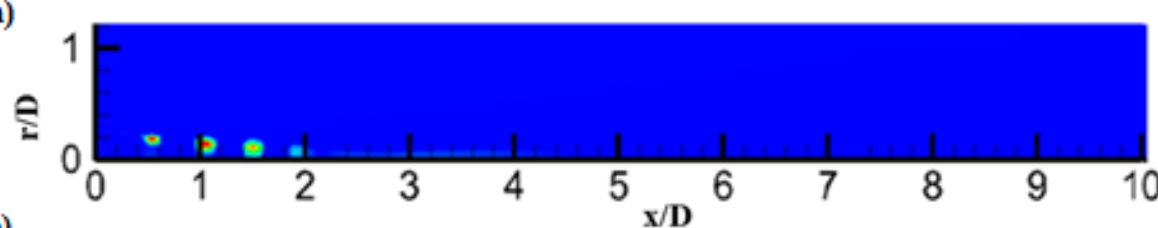

(b)

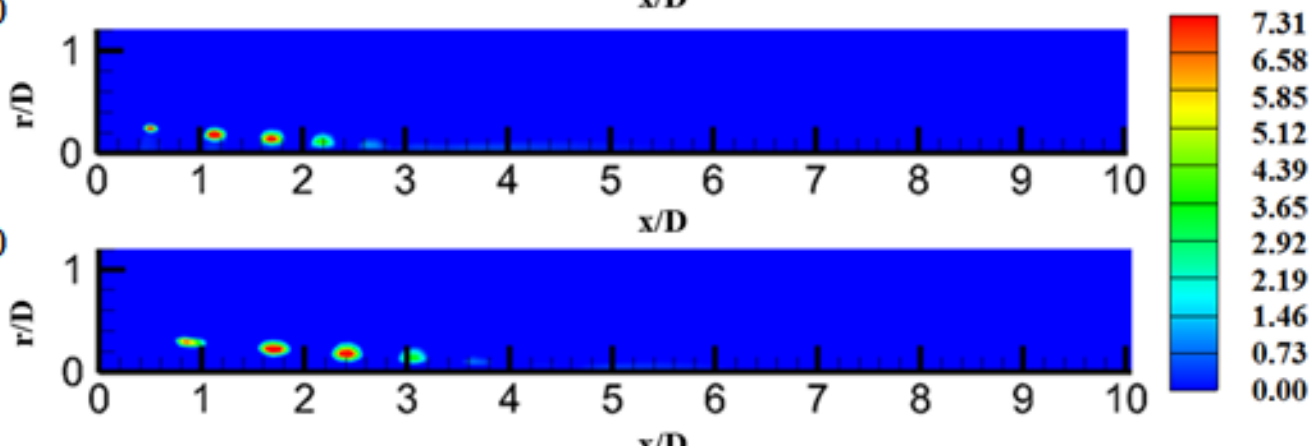

(d)

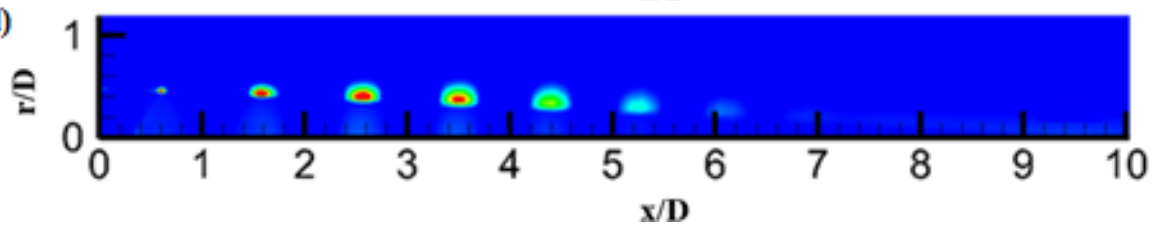

Fig. 18 Spatial distribution of acoustic source density for CD nozzles at (a)AR =3.0, (b) $A R=2.0$, (c) $\mathrm{AR}=1.5$, and (d) axisymmetric. 

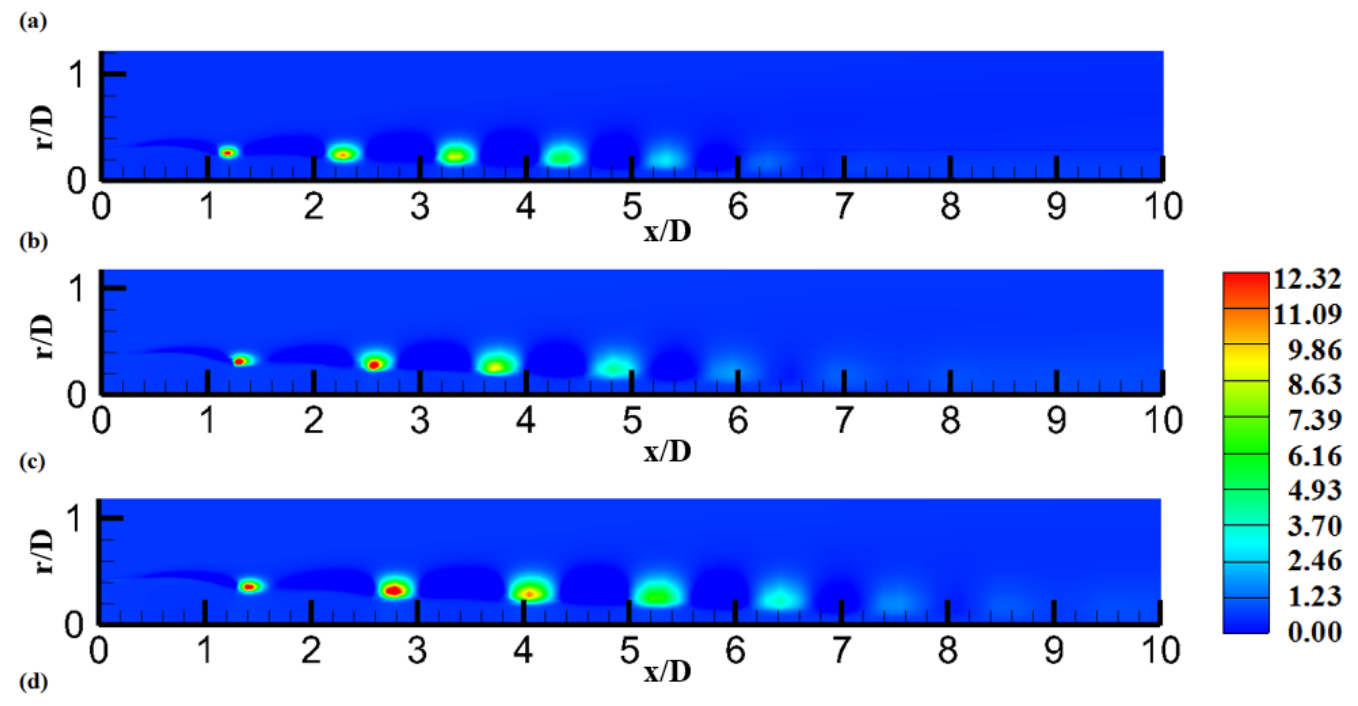

(d)

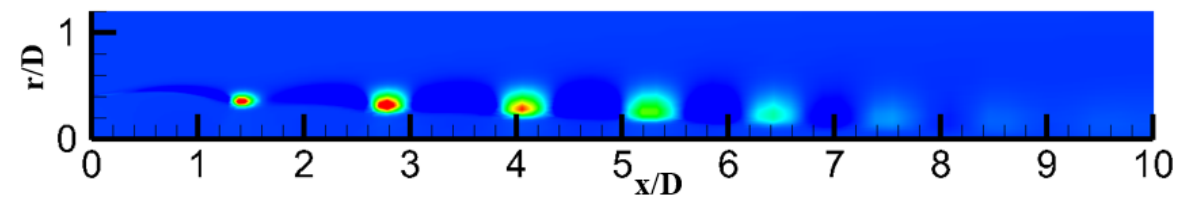

Fig. 19 Spatial distribution of acoustic source for convergent nozzles at (a)AR =3.0, (b) $A R=2.0$, (c)

$$
\mathrm{AR}=1.5 \text {, and (d) axisymmetric. }
$$

Figures 18 and 19 show the contours of the source density for the convergent-divergent and the convergent nozzles at $90^{\circ}$ observer location for various AR, respectively. On comparing the spatial source distribution for the jets of different $\mathrm{AR}$, it can be seen that as the aspect ratio increases, the size of the shockcell structure decreases and the distance between the peak sources reduces, while the most energetic source spots also reduce their intensity. This trend is especially noticeable for the convergent-divergent nozzles. The decrease of the effective source volume and the source amplitude should translate into the reduction of far-field noise levels with increasing AR.

For the far-field microphone location at 100D from the nozzle exit, fig. 20 and 21 show the acoustic spectra predictions for the jets of various $\mathrm{AR}$ and polar angles ranging from $80^{\circ}$ to $130^{\circ}$ for the overexpanded and the under-expanded nozzles, respectively. For each observer angle, the differences in the peak noise levels between the reference axisymmetric nozzle predictions with those of the asymmetric nozzle are listed on the same graphs. The experimental data corresponding to the baseline axisymmetric jet are provided in each case for comparison. As mentioned in section 4 , for the axisymmetric jets, the current 
mixed scale model captures the experimental data within a good range of most observer angles and frequencies $0.4<S t_{D}<3$ within $3 \mathrm{~dB}$.

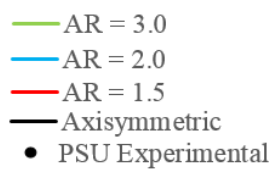

(a) $80^{\circ}$

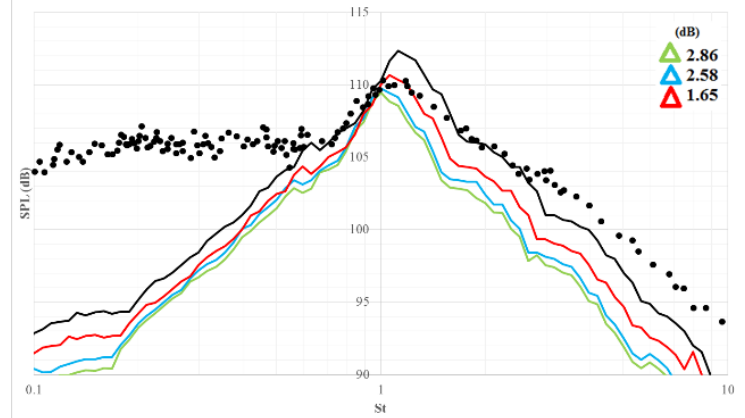

(c) $100^{0}$

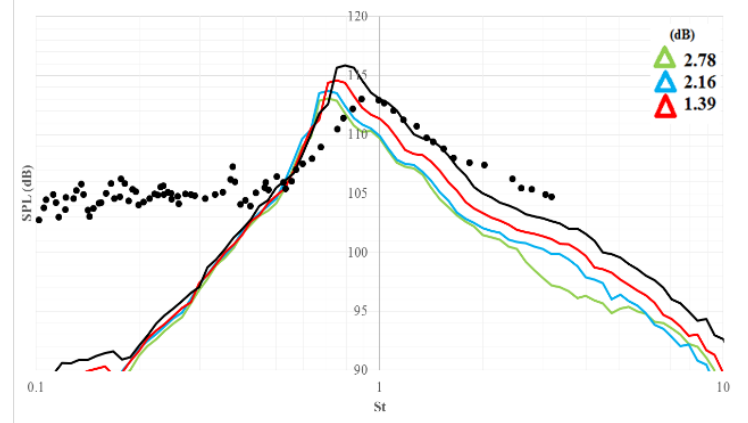

(e) $120^{\circ}$

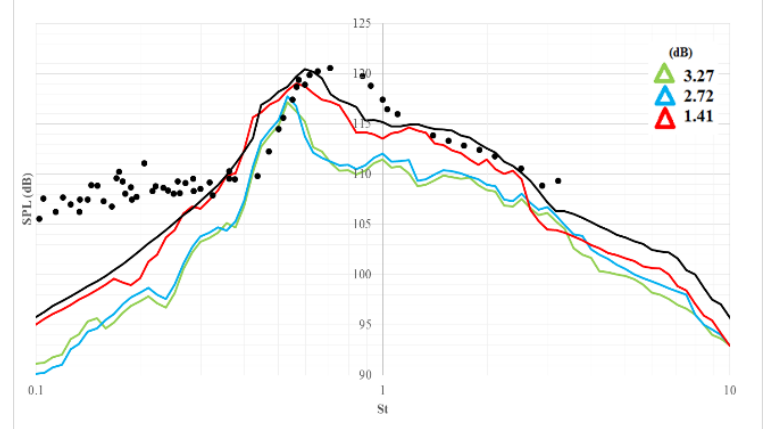

(b) $90^{\circ}$

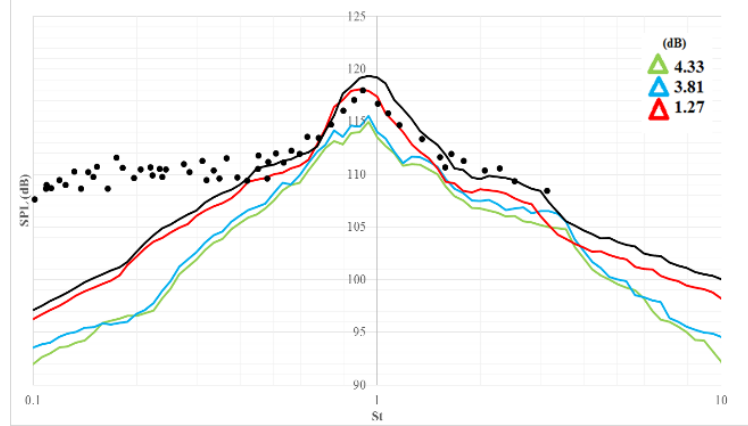

(d) $110^{0}$

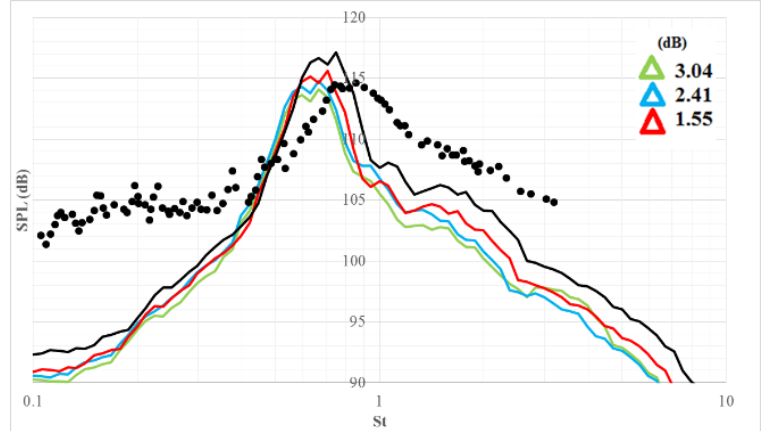

(f) $130^{\circ}$

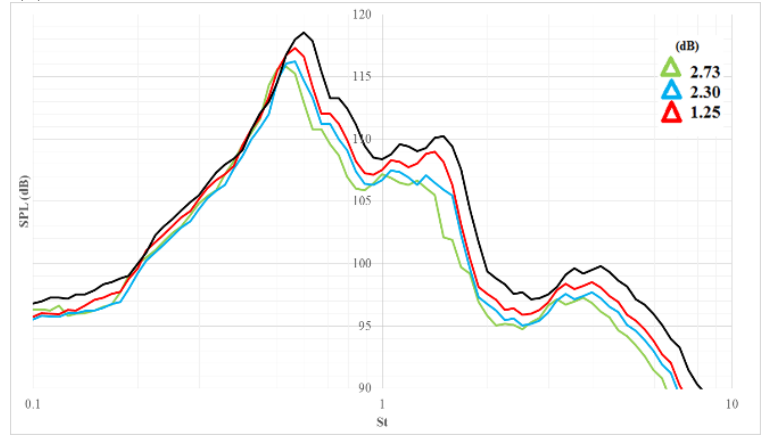

Fig. 20 Spectral predictions for CD axisymmetric and asymmetric nozzles. 
(a) $80^{\circ}$

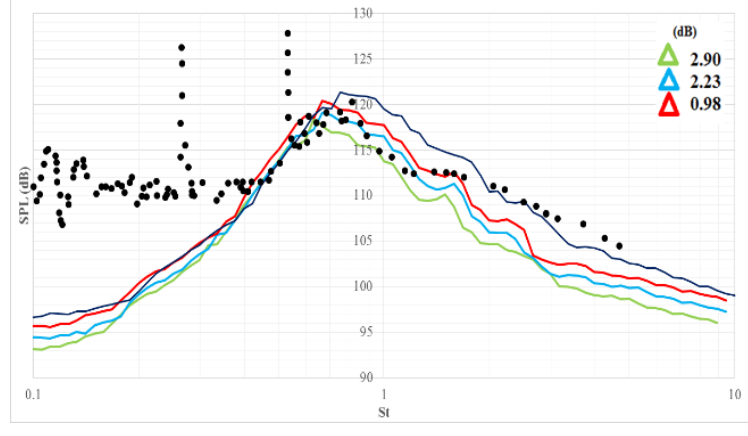

(c) $100^{0}$

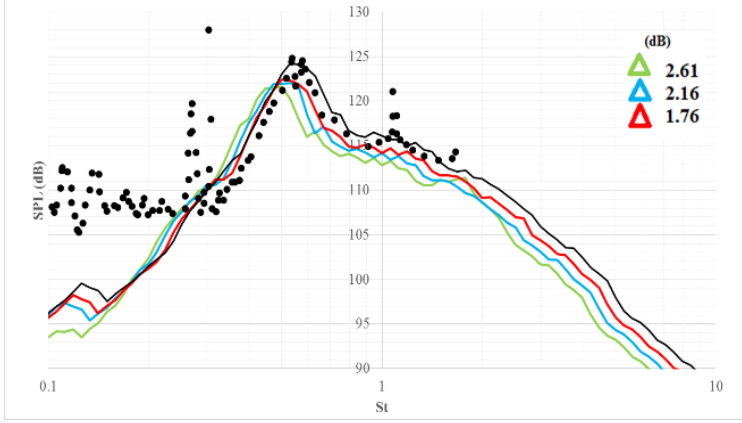

(e) $120^{\circ}$

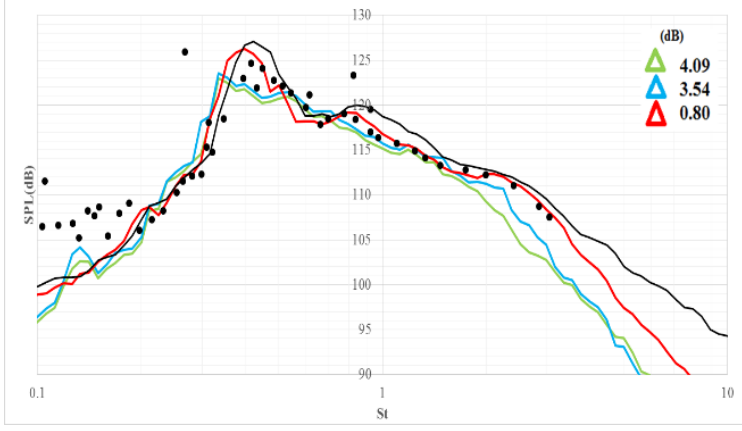

(b) $90^{\circ}$

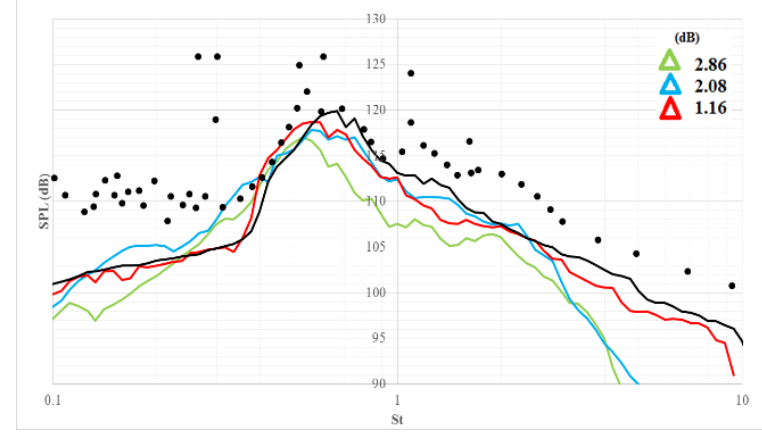

(d) $110^{0}$

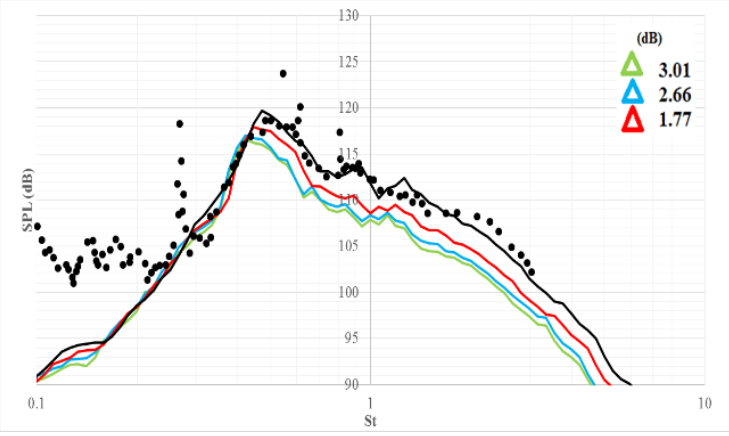

(f) $130^{\circ}$

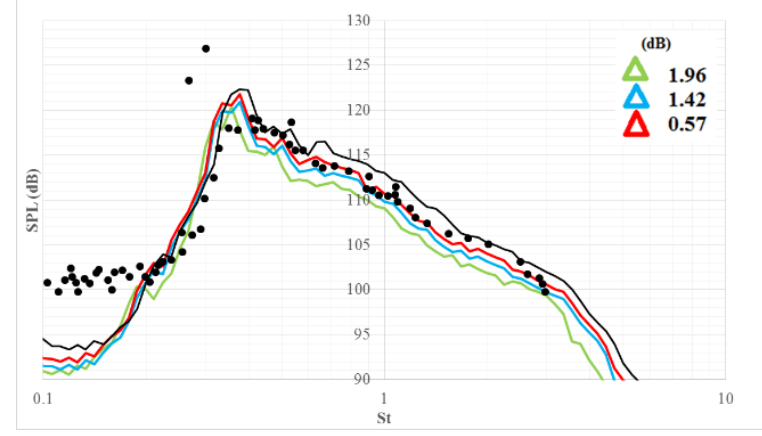

Fig. 21 Spectral predictions for convergent axisymmetric and asymmetric nozzles.

A clear trend emerges that marks a reduction in far-field noise peaks as the AR is increased. A reduction of at least $1.25 \mathrm{~dB}$ can be achieved with even a slightly elliptic nozzle in terms of the peak noise in the spectra. As expected from the comparison of correlation length scales and effective source distributions, the maximum reduction is observed for the elliptic nozzle corresponding to the largest $\mathrm{AR}=3.0$ for both 
nozzle types. For the convergent-divergent nozzle at $90^{\circ}$ observer angle, reductions of the peak of the noise spectra with respect to the axisymmetric case is as high as $4-5 \mathrm{~dB}$ for the nozzle having $\mathrm{AR}=3.0$.

Figures 22 and 23 show the OASPL directivity corresponding to BBSAN predictions for the same jet cases. As an acoustic model sensitivity check, OASPL predictions for the jets of varying eccentricity are compared for two models: the mixed scale model and the original Morris and Miller model based on the turbulence dissipation scale [6].

(a)

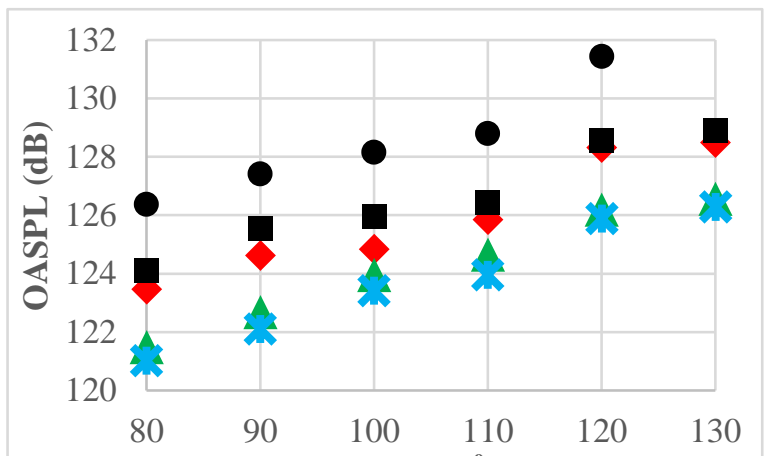

Angle $\left({ }^{0}\right)$
- PSU - Experimental

- Axisymmetric

$\bullet \mathrm{AR}=1.5$

$\triangle \mathrm{AR}=2.0$

$\mathbf{x} \mathrm{AR}=3.0$

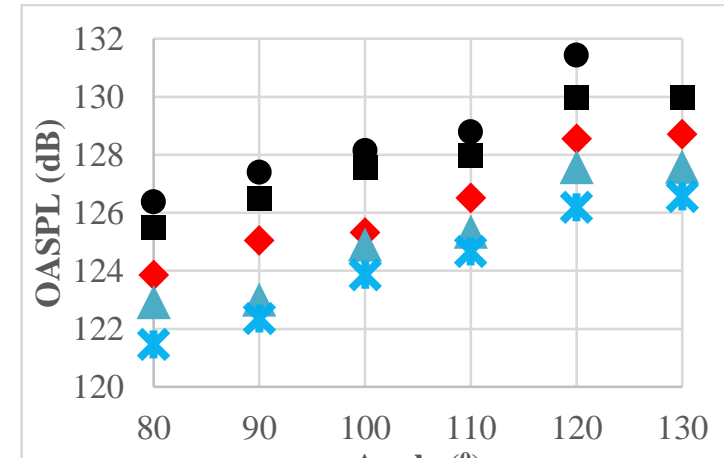

(b)

Fig. 22 OASPL directivity for CD nozzles (a) Morris and Miller Model [6]; (b) mixed scale model.

(a)

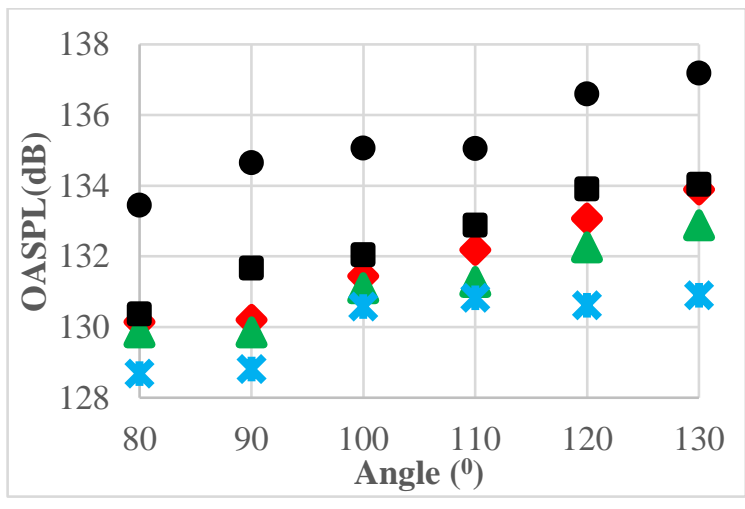

-PSU - Experimental

- Axisymmetric

$\rightarrow \mathrm{AR}=1.5$

$\triangle \mathrm{AR}=2.0$

$\mathbf{x} \mathrm{AR}=3.0$

(b)

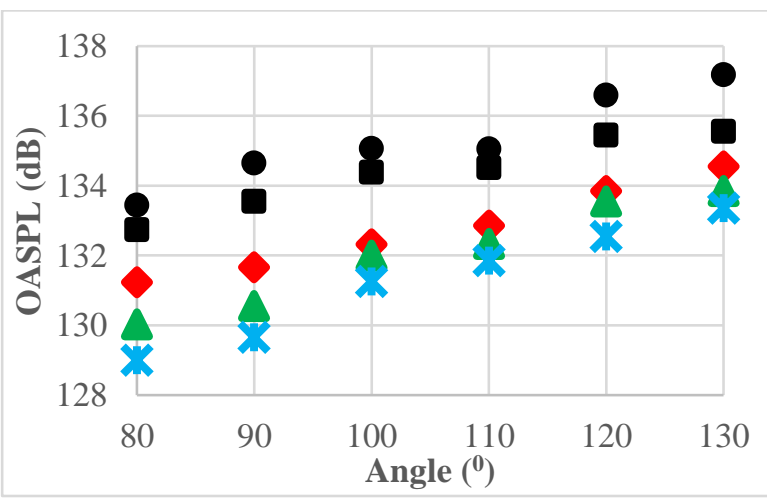

Fig. 23 OASPL directivity for convergent nozzles (a) Morris and Miller Model [6]; (b) mixed scale model. 
Both models predict the same general trend that the BBSAN decreases with increasing the jet aspect ratio and generally increases with the polar angle to the jet flow. The mixed scale model for the axisymmetric jets, which is in the best agreement with the experiments, predicts higher noise levels compared to the original Morris and Miller model [6]. On the other hand, the predictions of the two models for the highest aspect ratio elliptic jets, which correspond to the least prominent shock-cell structure, appear to be the same within $1 \mathrm{~dB}$ for most angles. A way to quantify the acoustic benefits of increasing AR is to consider the decrease of OASPL of the elliptic jets in comparison with the noise levels of the reference axisymmetric jets, i.e. through the relative noise level predictions. The relative noise predictions for various nozzle design modifications with respect to the baseline geometry are useful for elimination of possible systematic errors, which affect the accuracy of absolute predictions. Figures 24 and 25 show the corresponding relative OASPL directivity, $\triangle \mathrm{OASPL}$ for the mixed scale model and the original Morris and Miller model [6] in comparison with the axisymmetric jet predictions for each model.

Overall, the noise reduction predicted by both models for high aspect ratio jets and both nozzle types is comparable and is about $4-5 \mathrm{~dB}$. The most notable difference between the $\triangle \mathrm{OASPL}$ directivity results of the mixed scale model and those of the Morris and Miller [6] model is for the jets of small aspect ratio $\mathrm{AR}=1.5$, where the mixed scale model predicts a $1-2 \mathrm{~dB}$ reduction for most observer angles while the Morris and Miller model [6] shows only a marginal $0.5 \mathrm{~dB}-1 \mathrm{~dB}$ improvement. The difference between the predictions of the two acoustic models for the small AR jets and their similarity for the high AR jets is consistent with the previous discussion in section 4 about the differences between the total and the random part of the correlation length scales for axisymmetric jets. Indeed, for the jets with strong well-organised shock cells, which are typical of $\mathrm{AR}=1.5$ nozzle, the correlated part of the velocity fluctuations is an important part of the total fluctuations. This part of the velocity fluctuations can be referred to the process of turbulent eddy scattering by the shock cells that is explicitly accounted for in the mixed scale model but not in the original Morris and Miller model [6]. Once the shock cell strength reduces, which is the case for high AR jets, the random part of the velocity fluctuations associated with turbulent dissipation becomes 
most dominant. The latter is captured by the original Morris and Miller model [6] as well as by the mixed scale model which converge to the same in the limit of weak shock cells.

$\bullet \mathrm{AR}=1.5$

$\triangle \mathrm{AR}=2.0$

$\mathbf{x} \mathrm{AR}=3.0$

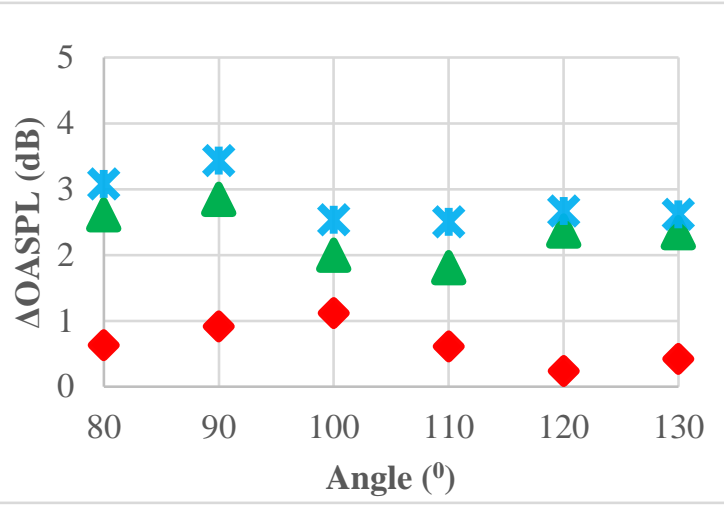

(b)

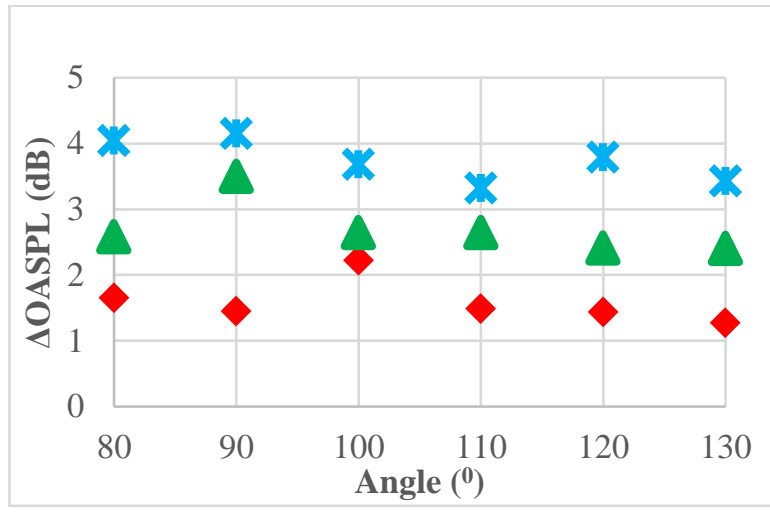

Fig. $1 \triangle$ OASPL directivity for CD nozzles using (a) Morris and Miller Model [5] and (b) mixed scale model.

$-\mathrm{AR}=1.5$

$\triangle \mathrm{AR}=2.0$

$\times \mathrm{AR}=3.0$

(a)

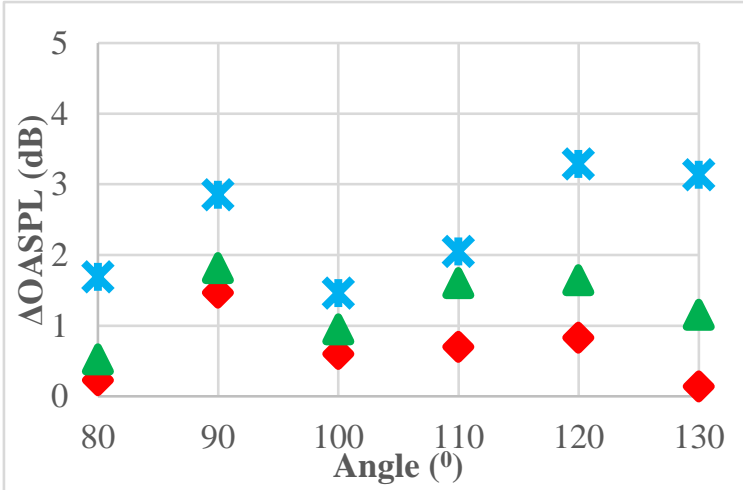

(b)

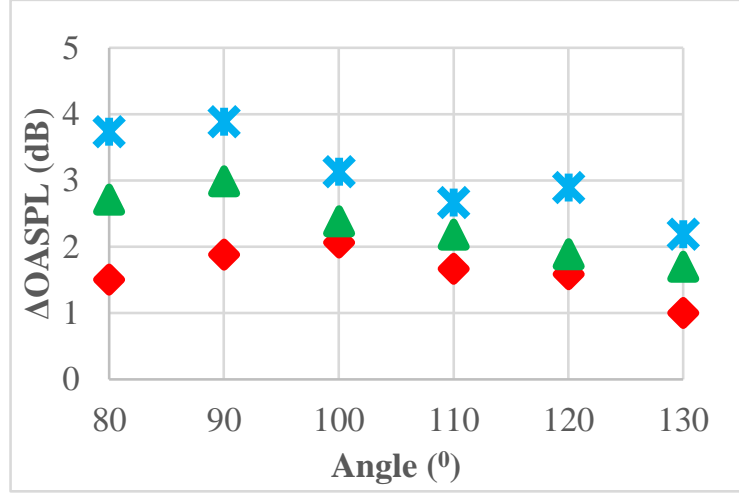

Fig. 2 $\triangle$ OASPL directivity for convergent nozzles using (a) Morris and Miller model [6] and (b) mixed scale model. 


\section{Conclusion and Discussion}

The Morris and Miller model of Broad Band Shock Associated Noise (BBSAN) [6] has been revisited to improve RANS-based sound predictions at high frequencies. Several modifications to the original model to incorporate the frequency-dependent length scales in the model based on recommendations from the literature have been considered. However, none of them resulted in any significant improvement over the original model. Then a new mixed scale model is proposed that incorporates the axial mean-flow velocity gradient in the definition of the correlation scale of the acoustic source. The motivation for this modification is to explicitly capture both the turbulence scattering by shock cells and the turbulence dissipation, which constitute two parts of the same BBSAN generation process, within the same acoustic analogy source. The modelling development has also been inspired by the recent high-resolution PIV experiment by Tan et al. [18] who provided an important experimental evidence for two distinct processes associated with supersonic jet noise: one is correlated and special for BBSAN and the other one is random and resembles the behaviour of perfectly expanded jets.

The resulting modification of the original Morris and Miller model is called the mixed scale model, which can be regarded as a unification of the original approach of Morris and Miller [6] and the two-scale model of Frendi et al. [20], which was originally proposed for jet mixing noise. In comparison with the original Morris and Miller model [6], the mixed scale model includes an additional calibration parameter which increases the model's accuracy at high frequencies. Similar to other model parameters, the additional calibration parameter can be determined from comparison of the model predictions with the far-field acoustic measurements for a chosen jet condition and observer angle.

The mean-flow solutions for two benchmark axisymmetric under-expanded and over-expanded jet cases have been obtained with a second-order Roe scheme in the framework of 3D RANS based on the twoequation $k-\omega$ SST model. A good agreement with the reference experimental data and numerical solution is reported for the total pressure and Mach profiles for the first several jet diameters. Using the RANS solutions obtained, several BBSAN models have been implemented including three modifications of the 
original Morris and Miller model [6] that incorporate frequency dependent scales and the new mixed scale model. For modelling of the benchmark axisymmetric jet cases, the following has been shown:

- the new mixed scale model reconstructs the acoustic correlation length scale distribution in the effective jet source that is in a good qualitative agreement with the integral correlation scales based on the total velocity fluctuations measured in the experiment of Tan et al. [18] for similar jet conditions; in comparison with this, the correlation length scale distributions predicted by the other models over the first 2-3 jet diameters show a more linear behaviour typical of the random part of velocity fluctuations driven by turbulent dissipation,

- the distribution of the effective source density in cylindrical-polar coordinates $(x, r)$ show multiple source patterns typical of the shock cell - turbulence interaction; at high frequency $\mathrm{St}_{\mathrm{D}}=2$, the effective sources are more pronounced for the new mixed scale model in comparison with the original Morris and Miller model [6], which provides the basis as to why the new model is expected to generate more noise at high frequencies,

- far-field noise predictions have been obtained for all modifications of the Morris and Miller model [6]; all acoustic models implemented predict the shift of the noise spectra peak to lower frequencies for a higher angle and capture the peak of the broadband spectra in comparison with the experiment within $2 \mathrm{~dB}$; apart from other things, this provides a 'safety check' that the current implementations of the baseline BBSAN models in this paper are correct,

- for higher frequencies, the original Morris and Miller model [6] and its modifications based on the frequency-dependent scales are broadly within $5 \mathrm{~dB}$ from the experiment for both the overexpanded and the under-expanded jet case; their Over-All Sound Pressure Level (OASPL) predictions are broadly within $2-3 \mathrm{~dB}$ from the experiment,

- the new mixed scale model captures high-frequency part of the spectra within $2 \mathrm{~dB}$ for the overexpanded jet case and within 3-4dB for the under-expanded jet for both $90^{\circ}$ and $120^{\circ}$ observer angle and frequencies $0.4<S t_{D}<3$ as compared to the experiment; for most other observer angles 
in the range between $80^{\circ}$ and $120^{\circ}$ the model predictions are within $3 \mathrm{~dB}$ from the experiment; the OASPL predictions of the mixed scale model are within $1 \mathrm{~dB}$ from the experiment, the sensitivity of the mixed scale model to the variation of calibration parameters within $+/-10 \%$ does not exceed $1 \mathrm{~dB}$,

- in comparison to the Tam model [4], which uses an empirical correction factor to account for the under-prediction at higher frequency, and the Harper-Bourne model [10], which uses a larger empirical dataset to account for the predictions at the higher frequencies, the mixed scale model presents an advantage with its improved higher frequency predictions without employing any additional empirical relationships,

Several elliptic jet modifications to the baseline axisymmetric convergent-divergent and convergent nozzles are considered by varying aspect ratio, $\mathrm{AR}=1.5,2$, and 3 for the same thrust conditions at the nozzle exit. Flow field solutions and far-field noise predictions are obtained. Acoustic sources and far-field sound predictions for the mixed scale model are analysed in detail. The noise spectra and the OASPL results are compared with predictions of the original Morris and Miller model [6] for the same jets. The following is demonstrated:

- for the axial distribution of the azimuthally averaged correlation length in the peak noise source region along the inner boundary of the shear layer, the magnitude of the correlation length scale decreases and its shape becomes more linear and smooth as the aspect ratio increases,

- as the aspect ratio increases, the effective source region of BBSAN reduces and the intensity of the multiple peak sources corresponding to the turbulence-shock cell interaction diminishes; altogether this leads to a decrease in the effective source volume as well as the source amplitude that explains why the elliptic nozzles become quieter with increasing their aspect ratio,

- for the peak noise spectra, a reduction of at least $1.2 \mathrm{~dB}$ can be achieved with even a slightly elliptic nozzle ( $\mathrm{AR}=1.5)$; for the convergent-divergent nozzle of $\mathrm{AR}=3$ at $90^{\circ}$ observer angle, 
reductions of the peak of the noise spectra with respect to the axisymmetric case is as large as $4-5 \mathrm{~dB}$,

- the comparison of the OASPL directivity predictions as well as the relative $\triangle$ OASPL predictions with respect to the baseline axisymmetric jets shows that an increase of the aspect ratio leads to a decrease in noise levels as predicted by the mixed scale model and the original Morris and Miller model [6]; both models suggest that the jets at the highest $A R=3.0$ are most beneficial in terms of noise reduction,

- the main difference between the mixed scale model and the original Morris and Miller model [6] for the predictions relative to the baseline axisymmetric jets, $\triangle$ OASPL concerning the noise reduction for jets of aspect ratio close to $1, A R=1.5$; the mixed scale model predicts a $1-2 \mathrm{~dB}$ reduction for most observer angles while the Morris and Miller model [6] shows only a marginal $0.5 \mathrm{~dB}-1 \mathrm{~dB}$ improvement for this case; these differences between the results of the two models are explained from the viewpoint of how the increased eccentricity of the elliptic jet changes the qualitative behaviour of BBSAN from a more correlated noise type associated with turbulence scattering by the shock cells to a less correlated process that is governed by turbulence dissipation; importantly, both of these processes are captured by the new mixed scale model.

Further work will include using high-resolution Particle Image Velocimetry and Large Eddy Simulation data for the direct evaluation of effective noise sources in supersonic jets. The high resolution source data can be useful to provide important insights for the development of RANS-based models of the second-order velocity auto-correlation functions and the corresponding anisotropic correlation scales for the refined modelling of BBSAN of elliptic jets. Additionally, further work will be devoted to implementation of more faithful sound propagation models to replace the current model based on the analytical free-space Green's function, which is limited to polar angles close to $90^{\circ}$ to the jet flow and neglects the variable sound speed effect on propagation that can be important in supersonic jets. 


\section{Acknowledgments}

The work has been partly supported by Queen Mary Innovation Fund. The authors are grateful to The School of Engineering and Materials Sciences, Queen Mary University of London for the computational resources used for this research and for the travel funding provided for the conference. S.A. Karabasov acknowledges the support of the Royal Society of London and Engineering and Physical Sciences Research Council (EPSRC), project number EP/I017747/1. The authors are also thankful to Dominic Tan from University of Monash for providing the integral length scale data plotted in fig. 1 and to the anonymous reviewers whose comments allowed to significantly improve the quality of the original manuscript.

\section{References}

[1] Bridges, J., Brown, C., Bozak, R., "Experiments on Exhaust Noise of Tightly Integrated Propulsion Systems", 20th AIAA/CEAS Aeroacoustics Conference, Atlanta, AIAA-2014-2904, 2014.

[2] Harper-Bourne, M. and M. J. Fisher, "The Noise from Shockwaves in Supersonic Jets," AGARD, 1973, pp. 1-13.

[3] Tam, C. K. W., and Tanna, H. K., “Shock-Associated Noise of Supersonic Jets from Convergent-Divergent nozzles,” Journal of Sound and Vibration, Vol. 81, No. 3, 1982, pp. 337-358.

[4] Tam, C. K. W., "Broadband Shock-Associated Noise of Moderately Imperfectly Expanded Supersonic Jets,” Journal of Sound and Vibration, Vol. 140, No. 1, 1990, pp. 55-71.

[5] D. C. Pack, “A note on Prandtl's formula for the wavelength of a supersonic gas jet,” Q. J. Mech. Appl. Math. 3, 173 , 1950.

[6] Morris, P. J. and Miller, S. A. E., "The Prediction of Broadband Shock-Associated Noise Using RANS CFD”, 15th AIAA/CEAS Aeroacoustics Conference (30th AIAA Aeroacoustics Conference), Miami, Florida, 2009.

[7] Lighthill M J., " On Sound Generated Aerodynamically. I. General theory". Proc. Roy. Soc. London Sen A 21, 11564-87, 1952.

[8] Tam, C.K.W. and Auriault, L., “Jet mixing noise from fine scale turbulence”, AIAA Journal, 22, No. 2, 145-153, 1999.

[9] Kalyan, A. and Karabasov, S.A., "Theoretical Modelling of Broadband Shock Associated Noise in Asymmetric Jets", 21st AIAA/CEAS Aeroacoustics Conference, Dallas, 2015, 2015-3127.

[10] Harper-Bourne, M, “Jet Near Field Noise Prediction”, 8th AIAA/ CEAS Conference, 2002- 2554.

[11] Self, R.H., "Jet Noise Prediction Using the Lighthill Acoustic Analogy”, Journal of Sound and Vibration, Vol 275, 2004, pp. $757-768$

[12] Self, R.H., and Bassetti, A., "A RANS Based Jet Noise Prediction Scheme”, 9th AIAA/CEAS Conference, South Carolina, 2013, 2003-3325. 
[13] Page, G.J., McGuirk, J.J., Hossain, M., Self, R.H., Bassetti, A., “A CFD Coupled Acoustics Approach for Coaxial Jet Noise”, 9th AIAA/CEAS Conference, South Carolina, 2013, 2003-3386.

[14] Morris, P.J. and Boluriaan, S., "The Prediction of Jet Noise from CFD Data," 10th AIAA/CEAS Aeroacoustics Conference, 2004-2977, 2004.

[15] Leib, S. and Goldstein, M. E., "Hybrid Source Model for Predicting High-Speed Jet Noise," AIAA Journal, Vol. 49, No. 7, 2011, pp. 1324-1335.

[16] Miller, S.A.E., "Towards a Comprehensive Model of Jet Noise using an Acoustic Analogy and Steady RANS Solutions", AIAA Journal, Vol. 52, Issue 10, 2013.

[17] Morris, P. J. and Zaman, K. B. M. Q., "Velocity Measurements in Jets with Application to Noise Source Modeling", Journal of Sound and Vibration, Vol. 329, No. 4, 2010, pp. 394-414.

[18] Tan, D.J., Soria, J., Honnery, D., Edgington-Mitchell, D., "A Novel Method for Decoupling the Velocity Fluctuations in Screeching Axisymmetric Jets.”, 22nd AIAA/CEAS Aeroacoustics Conference, Lyon, 2016, 2016- 2801.

[19] Fleury, V., Bailly, C., Jondeau, E., Michard, M., and Juv'e, D., "Space-time correlations in two subsonic jets using dual particle image velocimetry measurements," AIAA journal, Vol. 46, No. 10, 2008, pp. 2498-2509.

[20] Frendi, A., Dorland, W.D., Nesman, T., Wang, T.S., “A Jet Engine Noise Measurement and Prediction Tool”, Journal of Acoustical Society of America, 112(1), 2002, pp. 2036 - 2042.

[21] Ghahramani, S., Fundamentals of Probability (2nd Edition). Prentice Hall: New Jersey, 2000.

[22] Kalyan, A. and Karabasov, S.A., "Towards a Suitable Turbulence Length and Temporal Scale Model for Broadband Shock Associated Noise", 22nd AIAA/CEAS Aeroacoustics Conference, Lyon, 2016, 2016- 2802.

[23] Morris, P. J. and Miller, S. A. E., "The Prediction of Broadband Shock-Associated Noise Including Propagation Effects", International Journal of Aeroacoustics, Vol. 11, No. 7 \& 8, 2012, pp. 755-781.

[24] Miller, S. A. E., Veltin, J., Morris, P.J., McLaughlin, D. K., “Assessment of Computational Fluid Dynamics for Supersonic Shock Containing Jets”, AIAA Journal, Vol. 47., No.11, Nov. 2009.

[25] Wilcox, D. C., Turbulence Modeling for CFD, 3rd ed., DCW Industries, 2006.

[26] Pletcher, R.H., Tannehill, J.C. and Anderson, D.A., “Computational Fluid Mechanics and Heat Transfer”, 3 rd ed., CRC Press, 2013, pp. 7.

[27] Viswanathan, K, "Scaling Laws and a Method for Identifying Components of Jet Noise," AIAA Journal, 44(10), 2006, pp. 2274- 2285.

[28] Tam, C. K. W., "Stochastic Model Theory of Broadband Shock Associated Noise from Supersonic Jets," Journal of Sound and Vibration, Vol. 16, No. 2, 1987, pp. 265-302. 